NASA Technical Memorandum 100036

\title{
Measurements of the Free Stream Fluctuations Above a Turbulent Boundary Layer
}

David H. Wood,

Russell V. Westphal, Ames Research Center, Moffett Field, California

November 1987

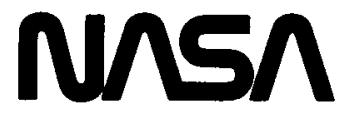

National Aeronautics and

Space Administration 


\section{SYMBOLS}

$\begin{array}{ll}C_{f} & \text { skin friction coefficient, } C_{f} \equiv \tau_{\text {wall }} /\left(1 / 2 \rho U_{e}^{2}\right) \\ f & \text { frequency }(\mathrm{Hz}) \\ R_{u v}, R_{u w} & \text { correlation coefficients, e.g. } R_{u v} \equiv \overline{u v} / \sqrt{\left(\overline{u^{2}}\right)\left(\overline{v^{2}}\right)} \\ R e_{\theta} & \text { momentum thickness Reynolds number, } R e_{\theta} \equiv U_{e} \theta / \nu \\ t & \text { time } \\ U_{\tau} & \text { friction velocity, } U_{\tau} \equiv \sqrt{\overline{\tau_{w a l l}} / \rho} \\ U, V, W & \text { mean velocities } \\ u, v, w & \text { fluctuating velocity components in } X, Y, Z \text { directions } \\ X, Y, Z & \text { right-hand Cartesian coordinate directions } \\ \delta^{*} & \text { boundary layer displacement thickness, } \delta^{*} \equiv \int_{0}^{\infty}\left(1-U / U_{e}\right) d Y \\ \delta_{99} & \text { boundary layer thickness, defined as } \delta_{99} \equiv Y\left(U / U_{e}=0.99\right) \\ \theta & \text { boundary layer momentum thickness, } \theta \equiv \int_{0}^{\infty} U / U_{e}\left(1-U / U_{e}\right) d Y \\ \nu & \text { air kinematic viscosity } \\ \rho & \text { air density } \\ \tau & \text { time increment } \\ \tau_{w a l l} & \text { skin friction } \\ \Phi & \text { power spectral density } \\ ()_{c} & \text { "corrected" component } \\ ()_{e} & \text { local free stream conditions } \\ ()_{i} & \text { turbulence-induced potential flow fluctuation contribution } \\ ()_{t} & \text { free stream turbulence contribution } \\ ()_{u} & \text { unsteadiness contribution } \\ ()_{1} \text { or }()_{2} & \text { one of the locations for covariance measurements }\end{array}$




\title{
MEASUREMENTS OF THE FREE STREAM FLUCTUATIONS ABOVE A TURBULENT BOUNDARY LAYER
}

\author{
David H. Wood and Russell V. Westphal
}

Ames Research Center

\section{SUMMARY}

This paper investigates the velocity fluctuations in the constant-pressure free stream above a turbulent boundary layer. It is proposed that the fluctuations receive contributions from three sources: (1) unsteadiness, (2) free stream turbulence, and (3) irrotational motion induced by the turbulent boundary layer. The three contributions to the total fluctuations are assumed to be statistically independent, and the unsteadiness is assumed to produce only axial motion. The magnitude of the unsteadiness was determined from values of the spatial covariance for separations large compared to the integral scale of the other contributions. Section 3 develops the theory of Phillips (1955) for the induced motion and provides a basis for determining the magnitude of this contribution. The free stream turbulence level was obtained by difference.

To test the model, experiments were conducted in a small blower-driven wind tunnel with a maximum boundary layer Reynolds number of $R e_{\theta}=5950$. The root-mean-square (r.m.s.) level of the axial fluctuations was about $0.2 \%$ of the mean free stream velocity. Measurements of all three Reynolds normal stresses, including their frequency spectra, were made with a crossed hot-wire anemometer, and two-point spatial covariance measurements were made using single hot wires. Normal stress measurements were made at four streamwise positions; covariance measurements were made in one streamwise plane only.

Although the small signal levels limited the accuracy of the measurements and necessitated the use of some judgement in determining the magnitudes of the various contributions, the proposed model did seem appropriate for decomposition of the fluctuations. It is argued that the technique of using spatial covariance measurements is preferable to the traditional alternative of high-pass filtering the anemometer signal to remove the unsteadiness. It is shown that the decomposition could be extended to the spectral densities of the contributions, if measurements of sufficient accuracy were available. An appendix contains suggestions for measurements in a free stream of low fluctuation level. 


\section{INTRODUCTION}

The fluctuations in the free stream bounding a turbulent shear layer can have a significant effect on that layer. As an extreme example, the breakdown to three-dimensionality in a mixing layer may well be controlled by the level of these fluctuations (Chandrasuda et al., 1978 and Wood \& Bradshaw, 1982). The nature of free stream disturbances can also determine the process of transition in laminar boundary layers, e.g. Saric (1986). One important practical consequence is the sensitivity to free stream conditions of airfoil lift and drag measurements in wind tunnel tests at low Reynolds number, e.g. Marchman (1987).

Bradshaw \& Pankhurst (1964), for example, divided the free stream fluctuations into two components: the turbulence, generated upstream of the working section, and the unsteadiness. The latter is usually associated with the low-frequency fluctuations consisting of the nearly axial motion produced by the fan or blower and the three dimensional (3-D) unsteadiness due to separation from a model or a badly designed component of the tunnel. In this work we will ignore the latter contribution, and define the unsteadiness to be the effectively one-dimensional (1-D) contribution to the free stream fluctuations. This definition, which allows the unsteadiness to be separated easily from the 3-D turbulence, should be adequate for the qualification of well-designed tunnels before model testing. For most boundary layer, or other shear layer tunnels, the present definition should always be adequate, provided the working-section dimensions do not alter significantly with the distance downstream. The effect of free stream turbulence and integral length scale on turbulent boundary layer development has been investigated by Hancock \& Bradshaw (1983), Blair (1983), and Castro (1984). Corresponding studies of unsteadiness include Jayaraman et al. (1982), Cook et al. (1985) and Coustiex (1986).

In general, free stream turbulence will influence boundary layer structure at much lower amplitudes than will unsteadiness, so it is necessary to separate the respective contributions to the free stream fluctuation level. The unsteadiness is often "removed" by high-pass filtering the anemometer output. Because the integral scale (of the autocorrelation) of the free stream turbulence can never be zero, this removal is incorrect in principle and should result in an underestimation of the true turbulence level. An alternative would be to assume isotropy of the free stream turbulence from which it follows that the difference between $\overline{v^{2}}$ or $\overline{w^{2}}$ and $\overline{u^{2}}$ is due to the unsteadiness. The accuracy of this assumption for the present flow will be discussed in Section 5.

Any turbulent shear layer bounding the free stream will induce an additional irrotational, but 3-D motion within that free stream. The induced motion, which for practical purposes extends about one shear layer thickness, $\delta_{99}$, from the mean edge of the layer, has been studied by Phillips (1955), Stewart (1956), and Wood \& Ferziger (1984, hereinafter cited as WF). The last-mentioned discuss the motion induced by two shear layers, such as on the top and bottom walls of a wind tunnel. The induced motion can contribute significantly to the free stream fluctuation levels only if $\delta_{99}$ is comparable to the height or width of the wind 
tunnel. This often occurs towards the end of boundary layer working sections and more rapidly in free-jet tunnels, Wood (1986).

The spectral distributions of the three contributions to the free stream fluctuations are shown qualitatively in figure 1. (Here, and throughout this paper, the spectral distributions, or densities, are defined so that their infinite integral with respect to frequency gives the mean square of the relevant velocity fluctuation.) At low frequency, the $u$-spectrum, $\Phi_{u u}$ (fig. 1a) receives contributions from the induced motion, as well as from the unsteadiness, so highpass filtering becomes even less desirable if the former is significant. Obviously, the integral scale of the unsteadiness is zero, as is that of the induced $u$-motion (see WF for details). The spectrum of the latter, $\Phi_{u u, i}$, increases approximately as frequency squared at low frequency (Bradshaw, 1967), and the position and value of the maximum spectral density depends on the distance from the shear layer (Bradshaw, 1967 and WF). The position of maximum $\Phi_{u u, u}$, the spectral density of the unsteadiness, is related to the blade passing frequency, while the shape of the turbulence spectrum, $\Phi_{u u, t}$, depends on the tunnel geometry and flow conditioning. The absence of unsteadiness in the $v$ - and $w$-components (fig. $1 \mathrm{~b}$ ) is partially offset by the non-zero integral scale of the induced motion (WF). Figure 1, and the identification of the many parameters that govern the contributions to the total free stream level, emphasize the difficulty of separating the contributions by high-pass filtering.

The main aim of the work described here is to explore methods of identifying and separating the three contributions to the total fluctuation levels in the free stream. Section 3 provides the necessary theoretical background by developing Phillips' (1955) theory for the induced fluctuations. Section 4 gives a description of the experiment and measuring techniques; unless stated otherwise, the techniques were those used routinely for measurements in boundary layers (where the fluctuation levels are much higher than in the free stream). The results are presented in Section 5, with emphasis on those from the furthest downstream station $(X=298 \mathrm{~cm})$ where the induced motion contributes significantly to the normal stresses near the boundary layer edge. At this location, the proposed separation was successful in allowing an estimate of the three contributions without resorting to highpass filtering. Section 6 contains concluding remarks, and the Appendix summarizes our experience in making measurements in the free stream with its inherently low fluctuation levels.

The present division of the free stream fluctuations may well be an over-simplification. For example, we will ignore acoustic effects, for the reasons given in Bradshaw (1967), and any interaction between the induced motion and the shear layer, which has been treated by Gartshore et al. (1983). Furthermore, we will assume that the mean flow is sufficiently two-dimensional (2-D) and uniform to prevent the generation of additional fluctuations via gradients in the mean velocity.

During the course of this work D.H.W. was supported by a Research Associateship from 
the National Research Council. The investigation of the relationship between the spectral densities was prompted by a discussion between D.H.W. and Prof. R.A. Antonia. The authors also thank Drs. S.S. Davis and R.D. Mehta for their comments and suggestions.

\section{THEORETICAL BACKGROUND}

The obvious generalization of figure 1 is to assume that the free stream fluctuations can be decomposed as follows

$$
u=u_{i}+u_{t}+u_{u}
$$

and

$$
v=v_{i}+v_{t}
$$

where the dependence on time (and position) is understood. The $w$-component equation is identical in form to that of equation (2). Assuming that the contributions to each component are independent, so that all cross-products such as $\overline{v_{i} v_{t}}$ are zero, gives

$$
\overline{u^{2}}=\overline{u_{i}^{2}}+\overline{u_{t}^{2}}+\overline{u_{u}^{2}}
$$

and similarly, but without the unsteadiness term, for the other two normal stresses. Furthermore, we will assume that the turbulence contributions are independent of $Y$. By definition, the unsteadiness is independent of both $X$ and $Y$.

The induced motion will be taken to obey the equations derived by Phillips (1955), so that

$$
\frac{1}{U_{e}^{2}}\left[\begin{array}{l}
\overline{u_{i}^{2}} \\
\frac{v_{i}^{2}}{w_{i}^{2}}
\end{array}\right]=\left[\begin{array}{l}
a_{u} \\
a_{v} \\
a_{w}
\end{array}\right]\left(\frac{Y-Y_{0}}{\delta^{*}}\right)^{-4},
$$

where $a_{u}, a_{w}$, and $a_{v}\left(=a_{u}+a_{w}\right)$ are constants and $Y_{0}$ is the effective origin for the induced motion. WF questioned the validity of Phillips' basic assumptions and hence, in principle, the accuracy of results like equation (4). However, a rigorous test of its vailidity appears to be precluded by the presence of the adjustable parameter $Y_{0}$, and the limited range over which the induced fluctuations are measurable. The normalization with $U_{e}$ and $\delta^{*}$ was used previously by Bradshaw (1967); its justification follows from the discussion of Stewart (1956) and WF. The relationship between the constants in equation (4) implies that 


$$
\overline{v_{i}^{2}}=\overline{u_{i}^{2}}+\overline{w_{i}^{2}}
$$

As pointed out by Stewart (1956), this equation is a consequence only of irrotationality, twodimensionality of the mean flow, and streamwise homogeneity. These conditions also give a relationship, which will be used later, between the spectra of the irrotational fluctuations. Consider the instantaneous motion at any time $t$, and at time $t+\tau$. By irrotationality

$$
u_{i}(t) \frac{\partial u_{i}(t+\tau)}{\partial Y}=u_{i}(t) \frac{\partial v_{i}(t+\tau)}{\partial X}
$$

Similarly

$$
u_{i}(t+\tau) \frac{\partial u_{i}(t)}{\partial Y}=u_{i}(t+\tau) \frac{\partial v_{i}(t)}{\partial X}
$$

Equations (6) and (7) can be summed to give an equation for $\partial\left(u_{i}(t) u_{i}(t+\tau)\right) / \partial Y$. Adding this to a similar equation for $\partial\left(w_{i}(t) w_{i}(t+\tau)\right) / \partial Y$, using continuity and time averaging gives the relationship between the time-delayed covariances as

$$
\begin{aligned}
& \frac{\partial}{\partial Y}\left[\overline{u_{i}(t) u_{i}(t+\tau)}+\overline{w_{i}(t) w_{i}(t+\tau)}-\overline{v_{i}(t) v_{i}(t+\tau)}\right] \\
& \quad=\frac{\partial}{\partial X}\left[\overline{u_{i}(t) v_{i}(t+\tau)}+\overline{v_{i}(t) u_{i}(t+\tau)}\right]+\frac{\partial}{\partial Z}\left[\overline{v_{i}(t) w_{i}(t+\tau)}+\overline{w_{i}(t) v_{i}(t+\tau)}\right] .
\end{aligned}
$$

Equation (8) for $\tau=0$ was derived by Corrsin \& Kistler (1955). If the streamwise, as well as spanwise, gradients of mean quantities can be ignored, and all covariances tend to zero as $Y \rightarrow \infty$ for all values of $\tau$, then equation (8) reduces to

$$
\overline{v_{i}(t) v_{i}(t+\tau)}=\overline{u_{i}(t) u_{i}(t+\tau)}+\overline{w_{i}(t) w_{i}(t+\tau)}
$$

which, for $\tau=0$, gives equation (5). Finally, by Fourier transforming (9), we have

$$
\Phi_{v v, i}(f)=\Phi_{u u, i}(f)+\Phi_{w w, i}(f)
$$

where, for example, $\Phi_{u u, i} \equiv \int_{0}^{\infty} \overline{u_{i}(t) u_{i}(t+\tau)} e^{i f \tau} d \tau$ is the spectral density of $\overline{u_{i}^{2}}$ at frequency $f$. (Hereinafter, the dependence of the spectral densities on $f$ will be understood.) Equation (10) was found by Antonia et al. (1987) to be in reasonable agreement with their measurements outside a plane wake. The equation is also an immediate consequence of Phillips' theory, but the derivation given here shows it to be independent of the particular assumptions made by Phillips about the effect of the turbulence on the potential flow. Similarly, equation (5) should be more general than (4). 


\section{EXPERIMENTAL APPARATUS AND PROCEDURES}

\subsection{Wind Tunnel}

The experiments were performed in the Boundary Layer Wind Tunnel located in the Fluid Mechanics Laboratory at Ames Research Center (fig. 2). A dust filter conditions the air entering the backward facing, airfoil-bladed blower. Flow conditioning upstream of the test section is acheived by a diffuser of area ratio 2.45 attached to the blower exhaust, followed by a settling chamber and a contraction. The contraction ratio is 6.0:1 in the vertical, $X-Y$ plane and $1.25: 1$ in the spanwise, $X-Z$ plane, so that the overall contraction ratio is 7.5. Coarse-mesh perforated plates of $64 \%$ open area are positioned at three locations for removal of total pressure nonuniformities that would otherwise occur after the blower, and for reduction in flow angularity preceding the honeycomb. Following the honeycomb are four identical 32 -mesh stainless steel screens $(62.9 \%$ open area) which are immediately upstream of the contraction.

The tunnel design produces a laminar boundary layer on the contraction walls entering the 20 by 80 by $300 \mathrm{~cm}$ test section. The end of the test section exhausts directly into the laboratory. At the free stream speed used for the present work, $20 \mathrm{~m} / \mathrm{s}$, the static pressure in the test section is maintained constant as measured by sidewall pressure tappings to about $0.5 \%$ of the free stream dynamic pressure by adjustment of the height of the control wall opposite the test surface. Therefore the height of the tunnel increased from $20 \mathrm{~cm}$ at the contaction end to about $21.3 \mathrm{~cm}$ at the working section outlet. A 0.4-mm-diam trip wire was positioned $20 \mathrm{~cm}$ from the contraction exit on both the upper and lower walls of the test section. The resulting boundary layer properties are summarized in table 1.

TABLE 1. - BOUNDARY LAYER PROPERTIES: $U_{e}=20 \mathrm{~m} / \mathrm{s}$.

\begin{tabular}{cccccc}
$X, \mathrm{~cm}$ & $\delta_{99}, \mathrm{~cm}$ & $\delta^{*}, \mathrm{~mm}$ & $R e_{\theta}$ & $C_{f}$ & $\delta^{*} / \theta$ \\
\hline \hline 95. & 1.4 & 2.25 & 2200. & 0.00355 & 1.41 \\
295. & 3.6 & 5.86 & 5950. & 0.00285 & 1.36
\end{tabular}

The most detailed measurements of flow quality were made over the center-half-span (40 $\mathrm{cm}$, about $15 \delta_{99}$ ) midway down the tunnel. The variation in $U_{e}$ was $1 / 4 \%$ with a maximum angularity of $1 / 4 \mathrm{deg}$. The corresponding variation in the wall shear stress was less than $8 \%$ (see fig. 15 of Westphal et al., 1987). This variation is considered exceptionally small (Mehta \& Hoffmann, 1987 give typical values), and further indicates the high quality of the mean free stream flow. 


\subsection{Hot-Wire Measurement Procedures}

Single, horizontal-sensor hot wires were used to measure the covariance of the streamwise velocity and $\mathrm{X}$-wires were used for all single-point measurements including spectra. The probes were constructed by the Ames Instrument Shop, and consisted of 1-mm-long, 5- $\mu \mathrm{m}-$ diam unplated Tungsten sensing elements soldered to support prongs. The spacing between wires of the $\mathrm{X}$-wire probes was about $1 \mathrm{~mm}$, and the wire angles were nominally $\pm 45^{\circ}$ with respect to the probe axis. The probes were operated with DISA (now DANTEK) 55M10 constant-temperature anemometers with a nominal overheat ratio of 0.8 . Square-wave tests were used to set the system frequency response above $10 \mathrm{kHz}$; the spectral content of all fluctuations to be measured was negligible above $1 \mathrm{kHz}$.

The hot-wire response equations and calibration methods used were as described by Westphal and Mehta (1984): a modified King's cooling law with cosine angular sensitivity was assumed, and calibration was performed at fixed angle and varying velocity combined with a yaw calibration at fixed velocity to determine the effective wire angles. The calibrations were performed in the free stream and the measurements were made with the plane of the wire sensors parallel to the $X-Y$ plane to obtain $u$ and $v$, and with the wires in the $X-Z$ plane to obtain $u$ and $w$.

A PDP 11/44 computer with a 12-bit analog-to-digital (A/D) converter was used for controlling the experiment and for data acquisition. Before conversion, a known DC voltage was subtracted from the hot wire bridge voltage signals - a process known as "bucking"which was then amplified by a factor of 10 or 20 to take full advantage of the $+/-5 \mathrm{~V}$ range of the converter. The sampling rates varied up to $2 \mathrm{kHz}$. The blower speed was controlled using the output of a D/A converter for automatic calibration and accurate setting of the experimental conditions. A stepper-motor traverse provided for movement of the $\mathrm{X}$-wire in the $Y$-direction, and for $Z$-direction movement of one of the probes used for two-point covariance measurements. Software used for calibration, experiment control, data acquisition and reduction, and off-line calculations with plotting and database management is described by Lichtenstein and Saunders (1983) and Hooper and Saunders (1985).

Only one alteration was made to that software. Immediately before the calculation of the velocity statistics, a known velocity, approximately equal to the mean, was subtracted from each velocity component. By reducing the effect of round-off errors, this significantly improved the accuracy of the velocity statistics for the very low fluctuation levels encountered in the free stream. Quantities such as $\overline{u^{2}}$ were obtained from $8 \mathrm{~K}$ total samples $(4,096$ per wire) collected over two seconds. The final results were always found as the average of 10 such measurements. The alternative, one measurement over a much longer time, was found to cause a spurious contribution to the turbulence statistics from the sensitivity of the hotwire calibrations to temperature variations in the Laboratory. The spectral densities of the normal stresses were obtained by ensemble-averaging 100 estimates, each resulting from a fast Fourier transform of $1 \mathrm{~K}(1,024)$ values of instantaneous velocity. The frequency increment 
(sampling rate divided by number of samples) varied between $\sim 0.5$ and $2.0 \mathrm{~Hz}$, and is given in the figure captions. The covariances were obtained by sampling each wire at $500 \mathrm{~Hz}$ for about $8 \mathrm{sec}$. The shorter averaging time was dictated mainly by the increased setting-up time between calibration and measurement. However, the correlation coefficient, which is the covariance divided by the two turbulence intensities, should converge more quickly than the covariance and could be used to reduce any scatter in those measurements.

\section{RESULTS AND DISCUSSION}

The downstream development of the normal stresses is shown in figures 3,4 , and 5 . Note that figures $3 \mathrm{a}$ and $\mathrm{b}$ contain the measurements of $\overline{u^{2}} / U_{e}^{2}$ obtained with the crossed-wire oriented in both the $X-Y$ and the $X-Z$ planes. At the outlet, the difference between the two values is significant when compared to the level of $\overline{u^{2}} / U_{e}^{2}$, but the difference tends to reduce downstream. The induced motion is primarily responsible for the large increases in all the normal stresses as the walls are approached. The exception is the odd behavior of $\overline{w^{2}} / U_{e}^{2}$ at the outlet, which is not matched by the other stresses, and for which we have no explanation. The induced motion is discussed in detail later, using additional measurements near the surface at $X=298 \mathrm{~cm}$. As expected, there is a large region at the outlet where the stresses are nearly constant, indicating that only the induced motion is strongly $Y$ dependent. As $X$ increases, this region narrows as a consequence of the thickening boundary layers, and hence the increasing importance of the induced motion, in agreement with the form of equation (4). There does not appear to be a systematic trend in the minimum values of any normal stress; it is possible, however, that there is a slight decrease in $\overline{u^{2}} / U_{e}^{2}$ and a concomitant rise in $\overline{v^{2}} / U_{e}^{2}$ and $\overline{w^{2}} / U_{e}^{2}$.

As pointed out by WF, streamwise homogeneity implies that $\overline{u v} / U_{e}^{2}$ is always zero in the induced flow, and hence the free stream. (It is obvious from equation (8) with the time delay $\tau=0$, that $\overline{u v}$ can be non-zero if the streamwise gradients are not negligible.) The other stress $(\overline{v w})$ appearing in the reduced form of equation (8) was not measured, but $\overline{u w}$, which was measured, can also be used to assess the two-dimensionality of the mean flow. The downstream development of the shear stresses is shown in figures 6 and 7 , in terms of the correlation coefficients, $R_{u v}$ and $R_{u w}$ respectively. The negative values of $R_{u v}$ at small $Y$ for the last streamwise measurement location are due to the boundary layer turbulence; $R_{u v}$ falls to around -0.45 close to the wall. As mentioned previously, the two-dimensionality of the mean velocity is particularly good in this tunnel, so the general magnitude of $R_{u w}$, around 0.1 , probably represents the accuracy to which $\overline{u w}$ can be measured at these low levels. The implication of the similar magnitude of $R_{u v}$ is that the flow is approximately homogeneous in the streamwise direction as might be expected for a zero pressure gradient boundary layer; the corresponding effects on $R_{u v}$ of the more rapidly growing single-stream mixing layer are documented by WF.

The free stream velocity spectra on the tunnel centerline are shown in figure 8 for $X=0$ 
and in figure 9 for $X=298 \mathrm{~cm}$. Comparison with the corresponding spectra of Tan-atichat $\&$ Harandi (1986) shows the influence of the many parameters that determine the fluctuation level. $\Phi_{u u}$ in figure 8 has a sharp peak at around $13 \mathrm{~Hz}$ that is present, to a much lesser extent, in $\Phi_{v v}$ but not in $\Phi_{w w}$. The analogous peak in figure 9 is present only in $\Phi_{u u}$ and will be shown later to be associated with the unsteadiness. The other spectra measured at 298 $\mathrm{cm}$ are shown in figures 10 to 12 in order of decreasing $Y$. Note that the apparent change in shape of the $13 \mathrm{~Hz}$ peak as $Y$ decreases is due partly to a change in the frequency increment. Equation (4) shows that the contribution of the induced motion to all spectra increases rapidly as $Y$ decreases, as do the frequencies at which the contributions occur (Bradshaw, 1967 and WF). Thus the induced motion causes the spectra to "fill out" as the boundary layer is approached. In addition, $\Phi_{w w}$ approaches $\Phi_{v v}$ at low frequency as predicted by Phillips' theory (see WF, equation 12).

The interaction between the free stream turbulence and unsteadiness and the $Y$-dependent induced motion is also shown clearly in figure 13 where the normal stresses at $298 \mathrm{~cm}$ are plotted so that they would collapse onto a straight line of positive slope if Phillips' theory was everywhere applicable. The induced motion is dominant only for $Y<6 \mathrm{~cm}(Y / \delta<1.5)$, approximately, after which $\overline{u^{2}}$ is greater than $\overline{v^{2}}$. The difference between the nearly coincident minimum levels of $\overline{v^{2}} / U_{e}^{2}$ and $\overline{w^{2}} / U_{e}^{2}$ and the minimum level of $\overline{u^{2}} / U_{e}^{2}$ is due mostly to the unsteadiness.

Because of the overlapping spectral densities of the turbulence and unsteadiness, the easiest way to determine the latter is from spatial covariance measurements. By our definition, the unsteadiness is the motion correlated over distances comparable to the working section size, and large compared to the shear layer thickness. Measurements of the covariance of $u$ over spanwise separations up to $36 \mathrm{~cm}(10 \delta)$ are shown in figure 14 for a range of $Y$. In this figure, $u_{1}$ and $u_{2}$ are the instantaneous velocities at points 1 and 2 , separated by distance $Z_{1}-Z_{2}$, and $\overline{u_{1} u_{2}}$ is the (spatial) covariance (at zero time delay). For $Y=5$ and $6 \mathrm{~cm}$, the large values of the covariance at small separation are caused by the induced motion, whose spatial structure was investigated by WF. At large separation, however, the measurements at all $Y$ tend to collapse, although there is considerable scatter because of the short averaging times used. The asymptotic value of $\overline{u_{1} u_{2}} / U_{e}^{2}$, as determined by averaging all results for $r>16 \mathrm{~cm}$, is $3.3 \times 10^{-6}$. This value, shown as the dashed line in figure 14 , is about two orders of magnitude greater than the correlated noise which was found as the covariance in still air with the offset and gain adjusted to give the usual "mean velocity". Because of the very large correlation scale of the unsteadiness, the asymptotic covariance should also be the contribution of the unsteadiness to (the single point) $\overline{u^{2}} / U_{e}^{2}$, that is,

$$
\lim _{r \rightarrow \infty} \overline{u_{1} u_{2}}=\overline{u_{u}^{2}}
$$

Using an analog spectrum analyser and correlator, several further measurements were made to ensure that both equation (11), and its underlying assumptions, are reasonable. Firstly, the high values of the coherence between $u_{1}$ and $u_{2}$ were concentrated around $13 \mathrm{~Hz}$, the 
frequency of the peak value of $\Phi_{u u}$ in figure 8a. Secondly, the space-time correlation at large time delay between $u_{1}$ and $u_{2}$ appeared to be an undamped $13 \mathrm{~Hz}$ sine wave with an amplitude of about $80 \%$ of the correlation at zero time delay. Finally, the peak in $\Phi_{u u}$ occurred at frequency that was proportional to the fan speed (and $U_{e}$ ). The only evidence against associating this maximum with a 1-D unsteadiness is the corresponding peak in $\Phi_{v v}$ at the contraction outlet (fig. $8 \mathrm{~b}$ ); this oddity is considered in the following paragraph.

Considering the flow through the contraction as inviscid and incompressible, then part of any irrotational, axial motion entering the contraction will be subsequently converted into $v$ motion, which lies in the direction of the larger contraction. Since the contraction is nearly 2$\mathrm{D}$, the $v$-motion must have the predominant frequency of the inlet flow. However, the tunnel centerline, along which the measurements in figures 6 and 7 were obtained, should ideally remain an instantaneous as well as a mean streamline. This suggests the possibility that the small peak in $\Phi_{v v}$ is due to a slight nonuniformity in the flow entering the contraction, or to slight geometric irregularity in the contraction shape. The much smaller contraction in the spanwise direction is, presumably, the reason that no significant $13 \mathrm{~Hz}$ peak appears in $\Phi_{w w}$ in figure 6. The $v$-component of any unsteadiness leaving the contraction will be strongly reduced in the constant-area working section further downstream. Thus the assumption that the unsteadiness is 1-D should become more accurate with increasing streamwise distance, provided of course that the working section does not change rapidly in size.

Returning to figure 3, the minimum value of $\overline{u^{2}} / U_{e}^{2}$ at $298 \mathrm{~cm}$, taken as the average over $8<Y<12(\mathrm{~cm})$ and from both planes, is $4.4 \times 10^{-6}$. Over the same range, the average difference in $\overline{u^{2}} / U_{\epsilon}^{2}$ from the two planes is $0.9 \times 10^{-6}$. The minimum values of $\overline{v^{2}} / U_{e}^{2}$ and $\overline{w^{2}} / U_{e}^{2}$ are approximately equal at $6.7 \times 10^{-7}$ (figs. 4 and 5). These minima should be due almost entirely to the turbulence and unsteadiness. They can be subtracted from the remaining measurements to form "corrected" values denoted by the subscript $c$. The corrected stresses should obey the theory of Section 3 and thereby allow an indirect assessment of the accuracy of the values for the unsteadiness and turbulence. Hopefully, any inaccuracy will be caused largely by a contribution from the induced motion to the minimum stresses. This would cause the correction to underestimate the induced stresses, and is the reason why we have introduced the subscript $c$,rather than continue to use $i$. The possibility of iteratively refining the minimum levels will be discussed later. The difference between the minimum values of $\overline{u^{2}}$ from the two measurement planes suggests the possibility that the correction should be different for each plane. However, any difference would complicate the task of estimating, say, the r.m.s. contributions to the total free stream fluctuation level, and it will be shown below that a plane-dependent correction is not justified in terms of equation (5).

The dashed lines fitted to the corrected stresses in figure 15 have the form required by equation (4), so that 


$$
\frac{1}{U_{e}^{2}}\left[\begin{array}{c}
\overline{u_{c}^{2}} \\
\frac{v_{c}^{2}}{w_{c}^{2}}
\end{array}\right]=\left[\begin{array}{l}
2.8 \times 10^{-3} \\
5.3 \times 10^{-3} \\
1.4 \times 10^{-3}
\end{array}\right]\left(\frac{Y}{\delta^{*}}-4.4\right)^{-4}
$$

Only the $X-Z$ plane measurements of $\overline{u^{2}}$ are shown as they appear to be the more accurate, for the reasons given below. The significance of the corrections is seen easily by a comparison with figure 11. The minimum levels are indicated by the horizontal arrows and the solid line is Bradshaw's (1967) fit to his $\overline{u_{c}^{2}} / U_{e}^{2}$ measurements. The agreement with the present results is reasonable, and suggests that equation (12) could be used to estimate the induced stresses if these have not been measured. It was hoped to use the results at $98 \mathrm{~cm}$ to test further the generality of equation (12), but this was precluded by the higher turbulence levels, and the narrower region of induced motion. Bradshaw (1967) showed that $a_{u}, a_{v}, a_{w}$ and $Y_{0}$ are strong functions of the pressure gradient. Thus equation (12) is likely to be applicable only to boundary layers with zero, or nearly zero, pressure gradients.

The correction to the normal stresses can also be assessed from the ratio $\left(\overline{u_{c}^{2}}+\overline{w_{c}^{2}}\right) / \overline{v_{c}^{2}}$ which, from equation (4), should be unity. The uncorrected ratio is generally high (fig. 16), and increases with $Y$ because of the unsteadiness. The corrected ratio is low for most $Y$ and for $\overline{u^{2}}$ from both planes, which suggests that employing a plane-dependent correction to $\overline{u^{2}}$ is not justified. The $X-Z$ plane measurements of $\overline{u^{2}}$ give the larger stress ratio, which is the reason why these results were plotted in figure 11. Figure 14 shows that the correction to $\overline{u^{2}} / U_{e}^{2}$ is too high and will lead to an overestimation of the free stream turbulence level, by an amount, however, that is likely to lie within the error of measurement. The low value of the stress ratio in figure 16 is reflected in the ratio $\left(a_{u}+a_{w}\right) / a_{v}$ from equation (12).

The minimum levels were determined by assuming that the induced motion was negligible near the tunnel centerline. Using equation (12), the largest induced normal stress, $\overline{v_{i}^{2}} / U_{t}^{2}$, is $2.0 \times 10^{-7}$ at $Y=10 \mathrm{~cm}$ and $1.2 \times 10^{-7}$ at $Y=12 \mathrm{~cm}$. By equation (10) of WF, these values should be nearly doubled to account for the upper wall boundary layer. The resulting values seem far too high, partly because the consequent reduction in the minimum level will reduce the stress ratio in figure 16 even further. We will therefore not revise the estimates for the minimum stresses; of course, any revision would require iteration between the estimated minimum levels and the corrected stresses. In terms of the commonly used percentage intensities, the estimates for the various components are: $u$-component combined unsteadiness and turbulence, $0.21 \%$; $u$-component turbulence, $0.10 \%$; unsteadiness, $0.18 \%$; $v$ - and $w$-component turbulence, $0.08 \%$. By assuming that the turbulence is isotropic, and so determining $\overline{u_{c}^{2}}$ as the difference between $\overline{u^{2}}$ and $\overline{v^{2}}$ or $\overline{w^{2}}$ changes the $u$-component turbulence to $0.08 \%$ and the unsteadiness to $0.19 \%$.

The theory in Section 3 can also be used to investigate the spectral distribution of the axial component of the free stream turbulence and unsteadiness. If it is reasonable to assume isotropy for the turbulence, then $\Phi_{w w, t}=\Phi_{v v, t}$, and equation (10) reduces to 


$$
\Phi_{u u}-\left(\Phi_{v v}-\Phi_{w w}\right)=\Phi_{u u, u}+\Phi_{u u, t} .
$$

The first and the second (bracketted) term on the left-hand side are shown in figure 17 for various $Y$; the results at $5 \mathrm{~cm}$ look very similar to those at $6 \mathrm{~cm}$ (fig. 17c). As expected, there is considerable scatter in the terms, particularly in the second which was plotted only if it was positive and so has the smaller range in figures $17 \mathrm{a}$ and $\mathrm{b}$.

In principle, $\Phi_{u u, u}$ could be found from the co-spectrum of $u_{1}$ and $u_{2}$ used to find the covariance. Together with equation (13) this would allow the spectral decomposition of the free stream fluctuations and give the quantitative features of figure 1. However, improved measurement accuracy would be required to justify such a decomposition.

\section{CONCLUDING REMARKS}

The results presented here show that with some judgement, it is possible to obtain reasonable estimates for the contributions to the velocity fluctuations in the free stream of a conventional wind tunnel of modern design.

The streamwise component of these fluctuations receives contributions from the unsteadiness, the free stream turbulence and any induced motion, while the vertical and spanwise components contain only the last two. These contributions have overlapping spectral densities, as shown in figure 1 , and are determined by a large range of parameters. In spite of this, the unsteadiness is usually removed by high-pass filtering, which should result in an underestimation of the turbulence level. As an alternative, the unsteadiness is defined here as the 1-D motion contributing to the free stream fluctuations. Thus it can be determined from spatial covariance measurements at separations large compared to the integral scales of the other contributions. The results show that this definition is a reasonable approximation, and the covariance measurements can be made with good accuracy.

If the free stream is bounded by a shear layer of comparable dimensions, the turbulence will induce a significant 3-D, but irrotational, contribution to the fluctuation levels. Section 5 describes a reasonably satisfactory separation of the induced motion from the unsteadiness and turbulence. The separation was based on the theory in Section 3, a combination of simple manipulations of the constraints of irrotationality, and the more restrictive analysis of Phillips (1955). The resulting equation for the induced normal stresses, equation (12), should be applicable to any boundary layer in nearly 2-D, constant-pressure flow.

The techniques described here should allow the determination of, say, the r.m.s. level of the three contributions to the total fluctuations in any free stream with good mean uniformity. It was also shown that, in principle, the separation of the contributions can be extended to include their spectral densities, although this would require more accuracy than we obtained from the measurement techniques used here. 


\section{APPENDIX: GUIDE FOR FREE STREAM MEASUREMENTS}

This appendix provides a guide for the measurement of free stream fluctuation levels in low-speed wind tunnels. This guide is meant to be general, therefore some of the points given below were not addressed in the main text of this paper. It is assumed that the uniformity and angularity of the mean flow have been checked and found satisfactory. This is essential because, for example, significant mean velocity gradients in the free stream will lead to the production of turbulence energy. Also, the comments are relevant only to the case of 1-D unsteadiness, such as would be expected in a well-designed wind tunnel without a model in the working section. The guide is given in terms of general rules, many of which are based on the anticipated (small) levels of the fluctuations, and the consequent difficulty of measuring them accurately.

1. Measurements should include all components. In most flows, such as that considered in the main text, the free stream turbulence will be approximately isotropic, that is $\overline{v^{2}}$, $\overline{w^{2}}$, and the turbulence contribution to $\overline{u^{2}}$ will be roughly equal. Thus a large discrepancy between the measured $\overline{u^{2}}$ and the other components indicates immediately the importance of the unsteadiness. Similarly a reasonable agreement between the levels of $\overline{v^{2}}$ and $\overline{w^{2}}$ can be regarded as a consistency check on the measurements. It is emphasised, however, that isotropy is only an approximation which may not be valid for all flows.

2. Measurements should be made at a number of positions. This will check if there is any (a) local production of turbulence energy by mean flow gradients, (b) $Y$-dependence of the turbulence, or (c) $X$-dependence in the measurements. Point (a) is self-explanatory, while a significant $Y$-dependence would suggest the presence of induced motion. By definition, the unsteadiness is independent of $X$, and the free stream turbulence usually decays slowly, so that measurements showing a strong dependence on $X$ are likely to be in error. The only possible exception to this statement occurs for measurements close enough to the contraction outlet for the effects of residual streamline curvature to be significant.

3. The anemometer signals should be directly digitized with no high-pass filtering. The free stream turbulence receives contributions from all frequencies, so any filtering will tend to underestimate its magnitude. Direct digitization is preferred because most, if not all, analog r.m.s. meters have a high-pass filter.

4. Data analysis requires special care. The computation of signal statistics from the digitized instantaneous signals can result in round-off errors when fluctuation levels are small, because of the finite word length used for digital representation of real numbers in computers. For the single-precision PDP-11/44, we found errors in the statistics of sine waves, of amplitude about $0.3 \%$ of the mean, when calculated without the software changes described in Section 4.2. Similar checks using small sine waves should be made on any system used for free stream measurements.

5. Reduce or remove identifiable sources of noise. Good practice commonly includes 
use of fully differential A/D converters, isolated instrument power and ground circuits, and low-drift analog signal conditioning.

6. Anemometer noise levels should be measured. The easiest way is probably to put each hot wire in a separate, small sealed container. It is reasonable to assume that the noise, which usually originates in the anemometer's main unit, is uncorrelated with the velocity fluctuations. An improved estimate for the actual fluctuation level can therefore be obtained by subtracting the mean square noise level from the measured mean square of the fluctuations. Low-pass filtering - using a cut-off frequency above any energy-containing frequency - may help to reduce the effect of electronic noise. No general rules can be given for the appropriate cut-off frequency. An analog spectrum analyser was used here to determine when the $u$-component spectrum disappeared into the background noise.

7. The equation for the induced normal stresses (eqn. 12 of the main text) is applicable only to constant-pressure boundary layers, and so cannot be used for all turbulent flows.

8. If more than one turbulent shear layer is forcing the induced motion, the effects on the normal stresses are additive (see WF). 


\section{REFERENCES}

Antonia, R. A.; Shah, D. A.; and Browne, L. W. B.: The Organized Motion Outside a Turbulent Wake. Phys. Fluids, vol. 30, 1987, p. 2040.

Blair, M. F.: Influence of Free Stream Turbulence on Turbulent Boundary Layer Heat Transfer and Mean Profile Development. Parts I and II. J. Heat Trans., vol. 105, 1983, p. 33 and p. 41.

Bradshaw, P.: Irrotational Fluctuations Near a Turbulent Boundary Layer. J. Fluid Mech., vol. 27, 1967, p. 209.

Bradshaw, P. and Pankhurst, R. C.: The Design of Low Speed Wind Tunnels. Prog. Aero. Sci., vol. 5, 1964, p. 1.

Castro, I. P.: Effects of Free Stream Turbulence on Low Reynolds Number Boundary Layers. J. Fluids Engr., vol. 106, 1984, p. 298.

Chandrasuda, C.; Mehta, R. D.; Weir, A. D.; and Bradshaw, P.: Effect of Free-stream Turbulence on Large Structure in Turbulent Mixing Layers. J. Fluid Mech., vol. 85, 1978, p.693.

Cook, W. J.; Murphy, J. D.; and Owen, F. K.: An Experimental and Computational Study of Turbulent Boundary Layers in Oscillating Flow. Proc. 5th Symp. Turb. Shear Flows, Cornell Univ., 1985, p. 18.13.

Corrsin, S.; and Kistler, A. L.: Free-Stream Boundaries of Turbulent Flow. NACA Report $1244,1955$.

Cousteix, J.: Three-Dimensional and Unsteady Boundary Layer Computations. Ann. Rev. Fluid Mech., vol. 18, 1986, p. 173.

Gartshore, I. S.; Durbin, P. A.; and Hunt, J. C. R.: The Production of Turbulent Stresses in a Shear Flow by Irrotational Motion. J. Fluid Mech., vol. 137, 1983, p. 307.

Hancock, P. E.; and Bradshaw, P.: The Effect of Free Stream Turbulence on Turbulent Boundary Layers. J. Fluids Engr., vol. 105, 1983, p. 284.

Hooper, C.; and Saunders, D.: FMDAS: Fluid Mechanics Data Acquisition System. Report 7104-307, no. 13, Informatics General Corp., Feb. 1985.

Jayaraman, R.; Parikh, P.; and Reynolds, W. C.: An Experimental Study of the Dynamics of an Unsteady Turbulent Boundary Layer. Report TF-18, Mech. Eng. Dept, Stanford Univ., 1982. 
Lichtenstein, T.; and Saunders, D.: Data Management System for Mid-sized Data Acquisition and Display Systems. Report 7104-307, no. 11, Informatics General Corp., Dec. 1983.

Marchman, J. F.: Aerodynamic Testing at Low Reynolds Numbers. J. Aircraft, vol. 24, 1987, p. 107.

Mehta, R. D.; and Hoffmann, P. H.: Boundary Layer Two-Dimensionality on Wind Tunnel Walls. Expts. in Fluids, vol. 5, no. 5 May 1987, p. 358 .

Phillips, O. M.: The Irrotational Motion Outside a Free Turbulent Boundary. Proc. Camb. Phil. Soc., vol. 51, 1955, p. 220.

Raghunathan, S.; and McAdam, R. J. W.: Freestream Turbulence Effects on Attached Subsonic Turbulent Boundary Layers. AIAA J., vol. 21, 1983, p. 503.

Saric, W. S.: Boundary-Layer Transition to Turbulence: The Last Five Years. Proceedings of the Tenth Symposium on Turbulence, Univ. of Missouri, Rolla, Missouri, 1986, pp. 19-1:19-18.

Stewart, R. W.: Irrotational Motion Associated with Free Turbulent Flows. J. Fluid Mech., vol. 1, 1956, p. 593.

Tan-atichat, J.; and Hirandi, S.: Effects of Acoustic Disturbances on Measured Flow Characteristics Through a Contraction. AIAA J., vol. 25, May 1987, p. 659.

Westphal, R. V.; and Mehta, R. D.: Crossed Hot-Wire Anemometry Data Acquisition and Reduction System. NASA TM-85871, Jan. 1984.

Westphal, R. V.; Pauley, W. R.; and Eaton, J. K.: Interaction Between a Vortex and a Turbulent Boundary Layer. NASA TM-88361, Jan. 1987.

Wood, D. H.: A Note on Free-stream "Turbulence" Levels in Open-Jet Wind Tunnels. Wind Engg, vol. 10, 1986, p. 147.

Wood, D. H.; and Bradshaw, P.: A Turbulent Mixing Layer Constrained by a Solid Surface. Part 1. J. Fluid Mech., vol. 122, 1982, p.57.

Wood, D. H.; and Ferziger, J. H.: The Potential Flow Bounded by a Mixing Layer and a Solid Surface. Proc. Roy. Soc. vol. A395, 1984, p. 265. 

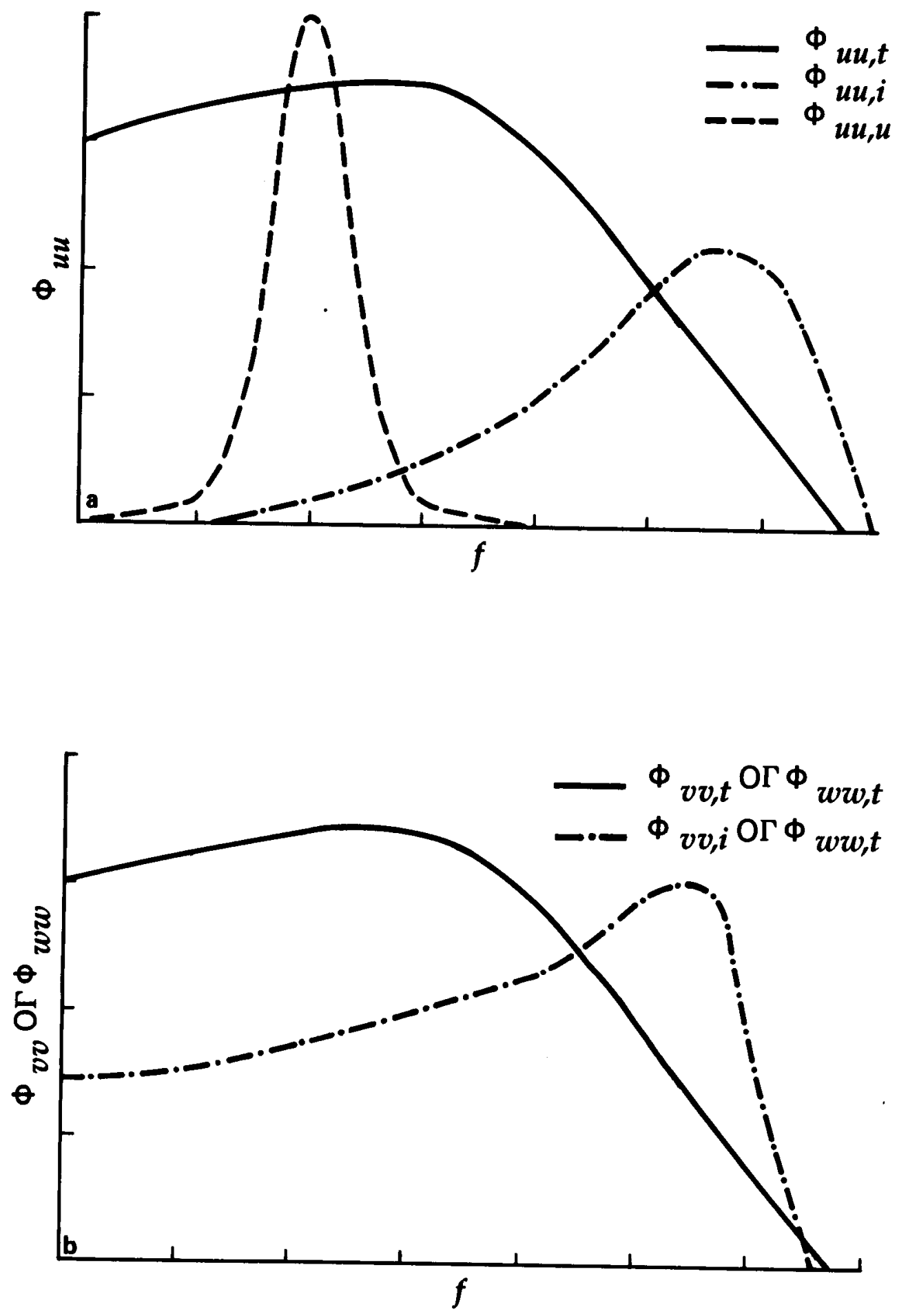

Figure 1. - Qualitative representation of spectral content of velocity fluctuations above a turbulent boundary layer. (a) $\overline{u^{2}} / U_{e}^{2}$. (b) $\overline{v^{2}} / U_{e}^{2}$ or $\overline{w^{2}} / U_{e}^{2}$. 


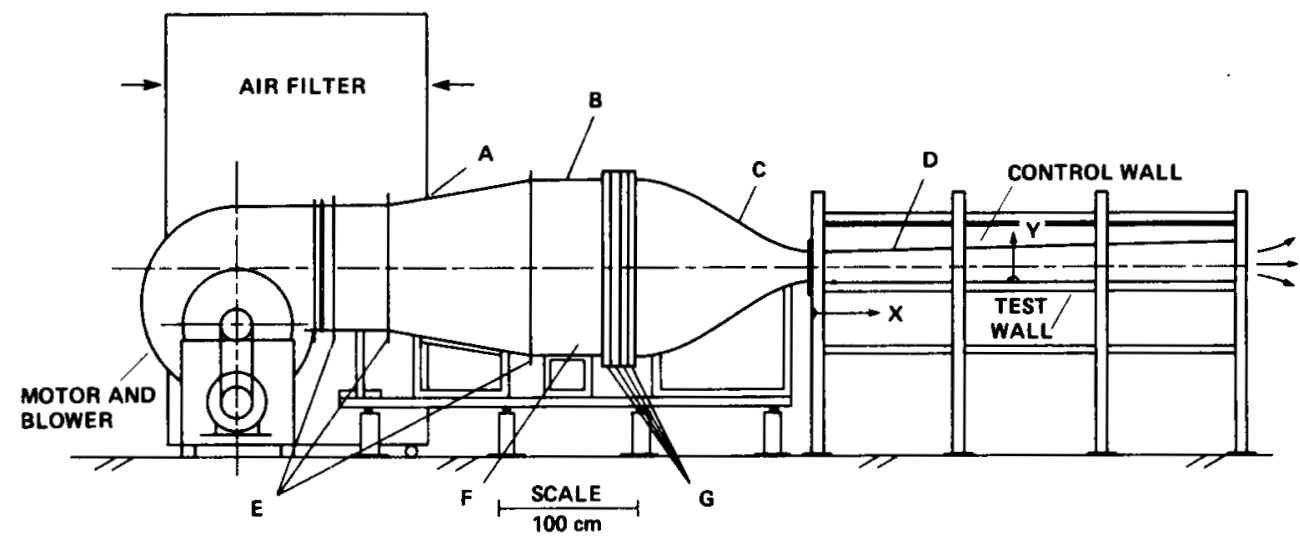

Part Description ${ }^{a}$
A. Diffuser
Area ratio: 2.45
Length: $0.81 d$,
B. Settling chamber
100 by $120\left(A_{s}=12000 \mathrm{~cm}^{2}\right)$ rectangular
Length: $0.40 d_{s}$
C. Contraction
Area ratio: 7.5
Length: $0.97 d_{s}$

Contours: symmetric $5^{\text {th }}$-order polynomials

with zero end slope and curvature

D. Test section

Height, width, length: 20 by 80 by 300

Adjustable control wall opposite test plate

E. Grids (three)

Open area: $64 \%$

Material: perforated steel plate, 0.16 thickness

Perforations: 1.27 square, 1.59 center-to-center

F. Honeycomb

Material: 0.0025 wall thickness, Aluminum

Cell size: 0.476 hexagonal-section

Cell length: 3.8

G. Screens (four)

Open area: $62.9 \%$

Material: 0.0114 stainless steel wire, 32 mesh

\footnotetext{
"lengths are given in $\mathrm{cm}$ or are dimensionless; $d_{*}$ is the equivalent settling chamber diameter: $d_{s}=\sqrt{4 A_{s} / \pi}=123.6$
}

Figure 2. - Boundary layer wind tunnel schematic and component description. 

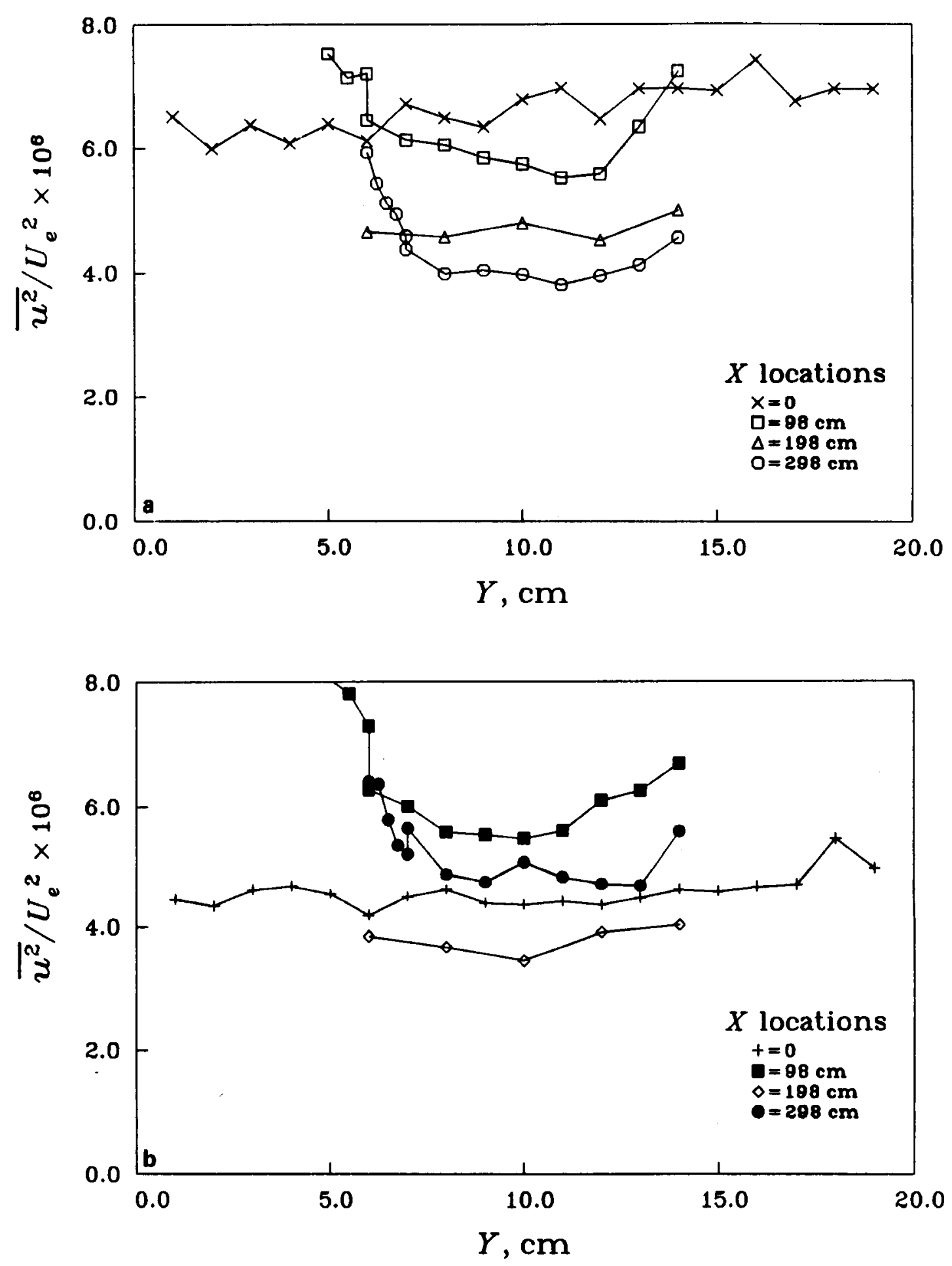

Figure 3. - Streamwise normal stress $\left(\overline{u^{2}} / U_{e}^{2}\right)$ outside the boundary layer at the four measurement locations. (a) $X-Y$ plane wire orientation. (b) $X-Z$ plane wire orientation. 


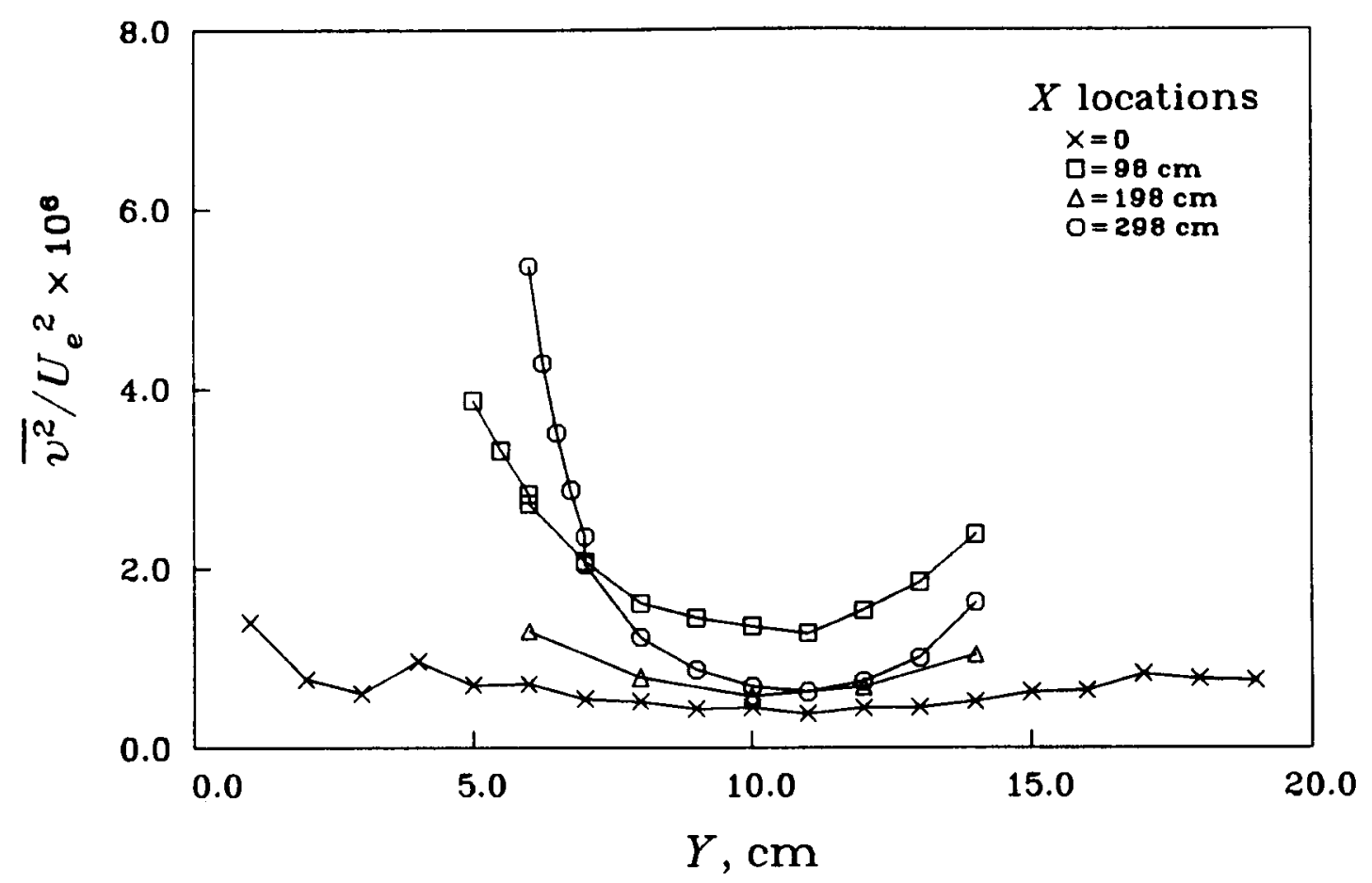

Figure 4. - Vertical normal stress $\left(\overline{v^{2}} / U_{e}^{2}\right)$ outside the boundary layer at the four measurement locations.

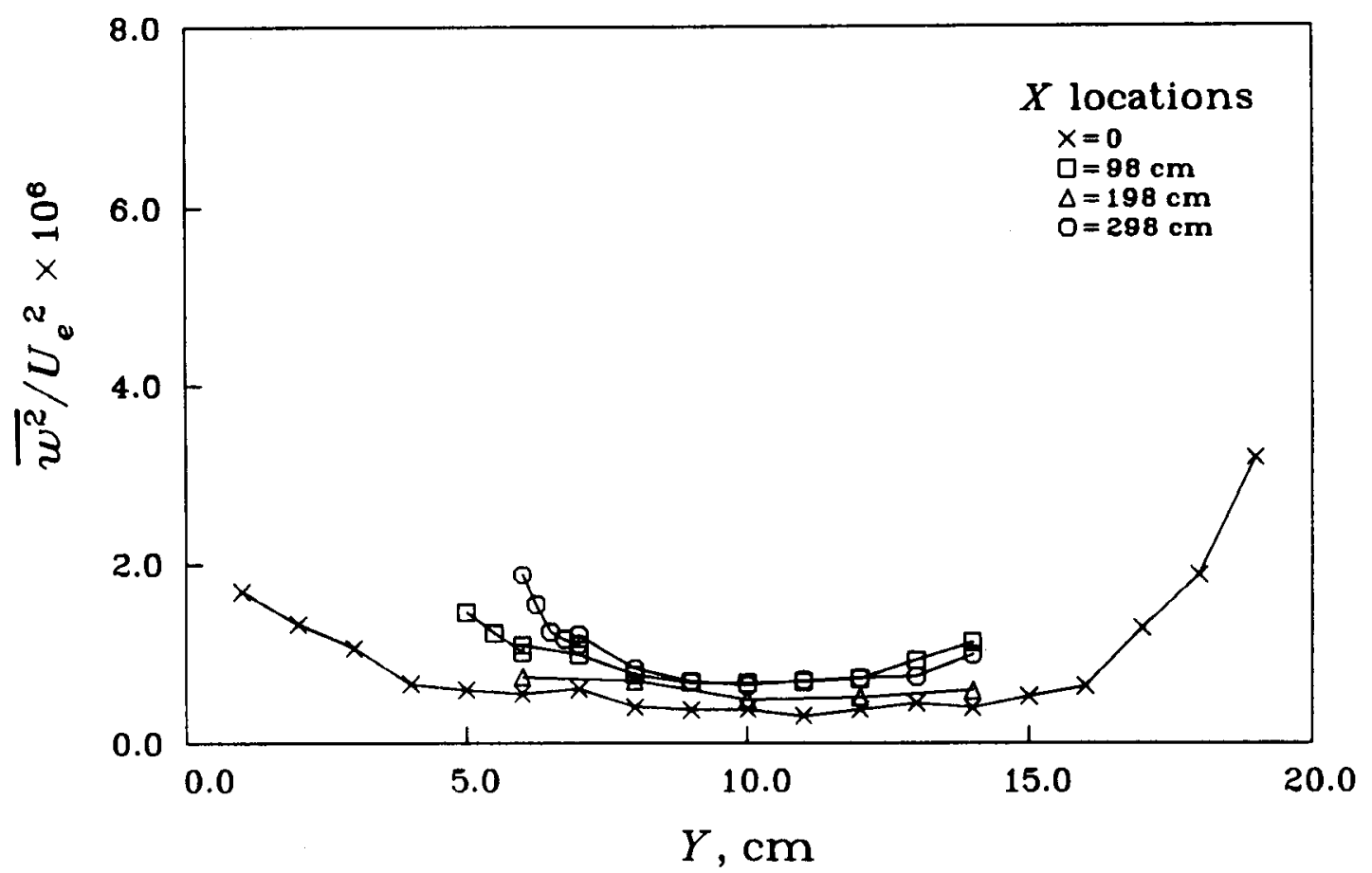

Figure 5. - Spanwise normal stress $\left(\overline{w^{2}} / U_{e}^{2}\right)$ outside the boundary layer at the four measurement locations. 


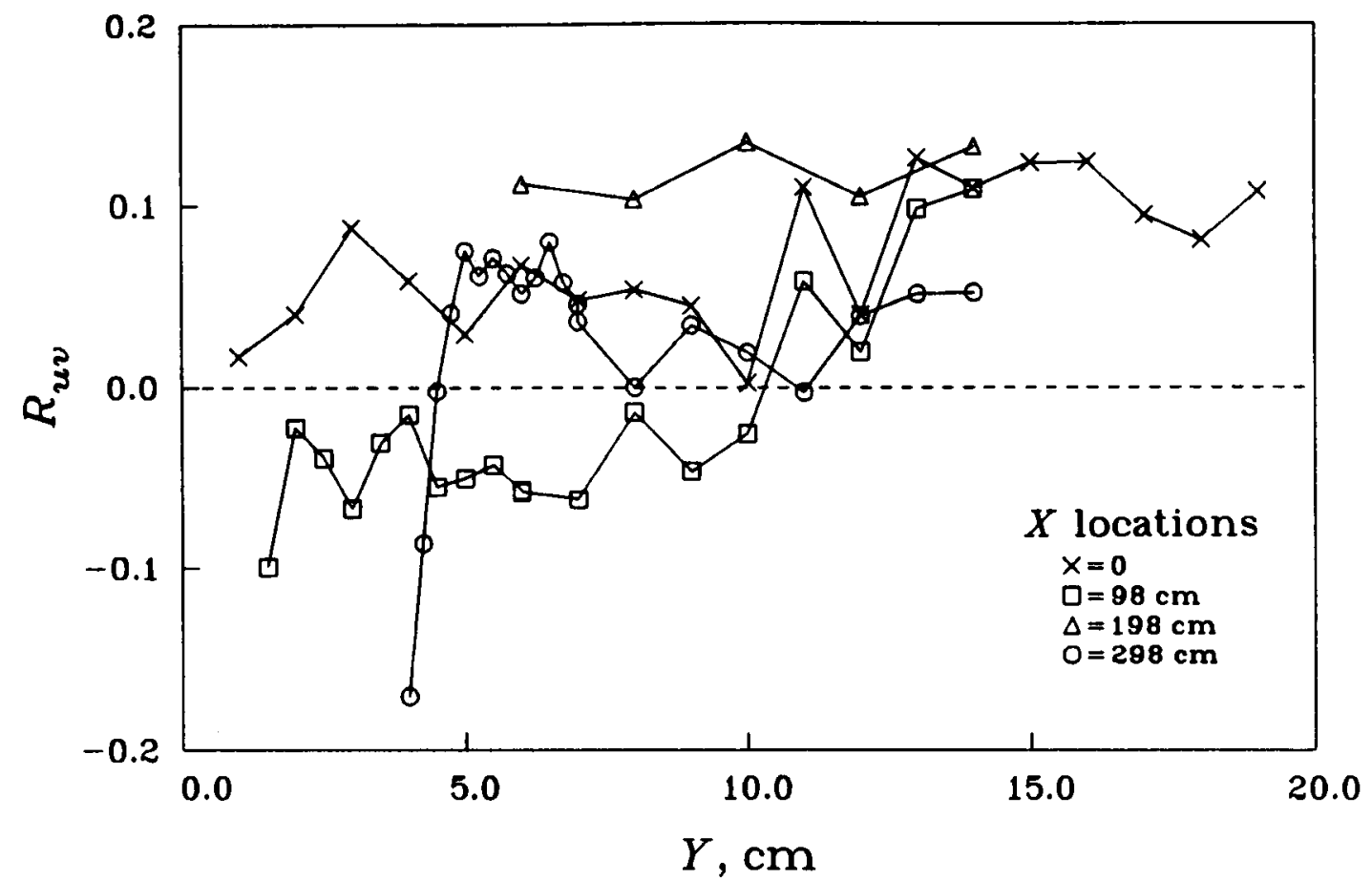

Figure 6. - Distributions of the correlation coefficient $R_{u v}$ outside the boundary layer at the four measurement locations.

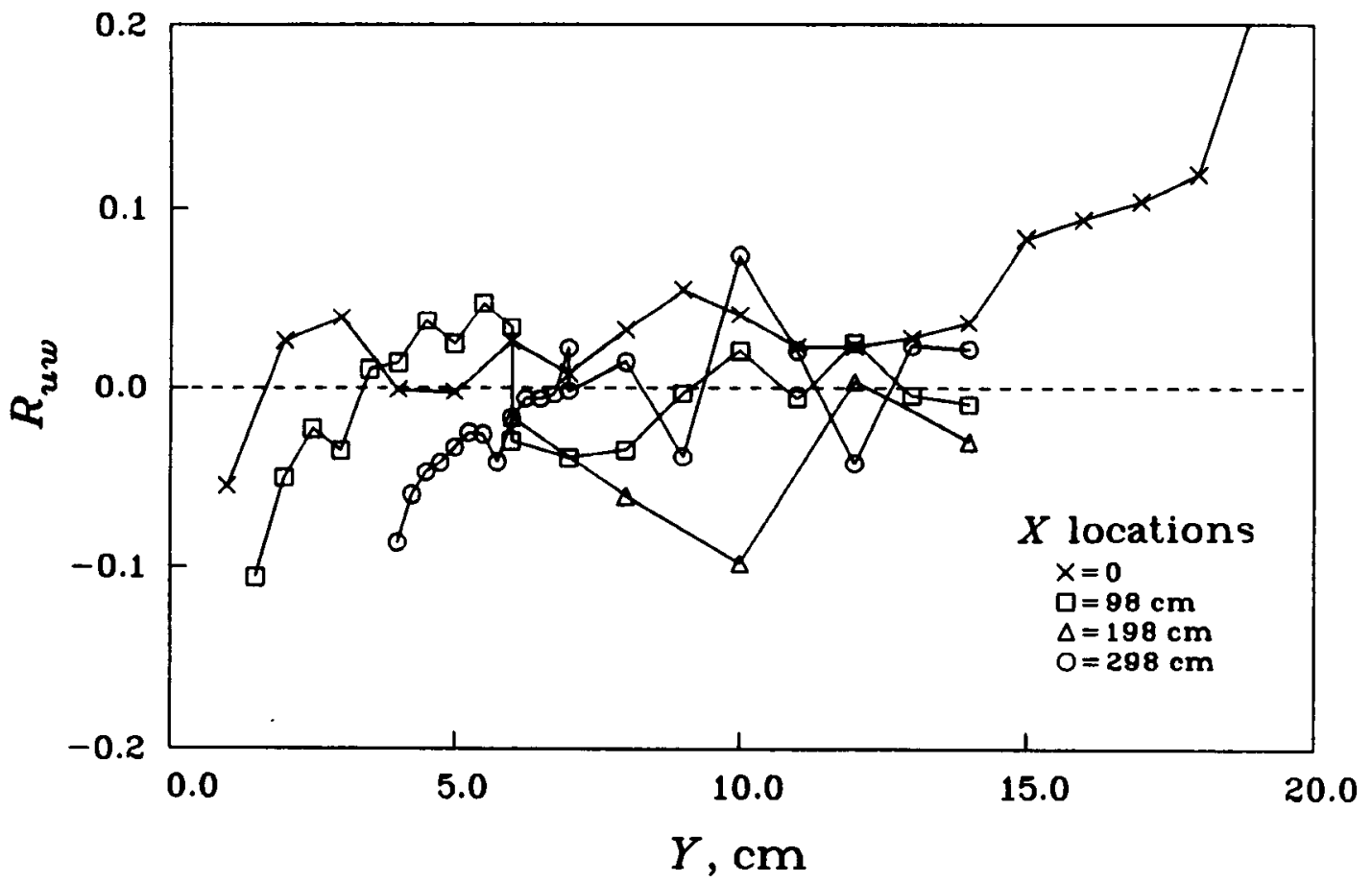

Figure 7. - Distributions of the correlation coefficient $R_{u w}$ outside the boundary layer at the four measurement locations. 


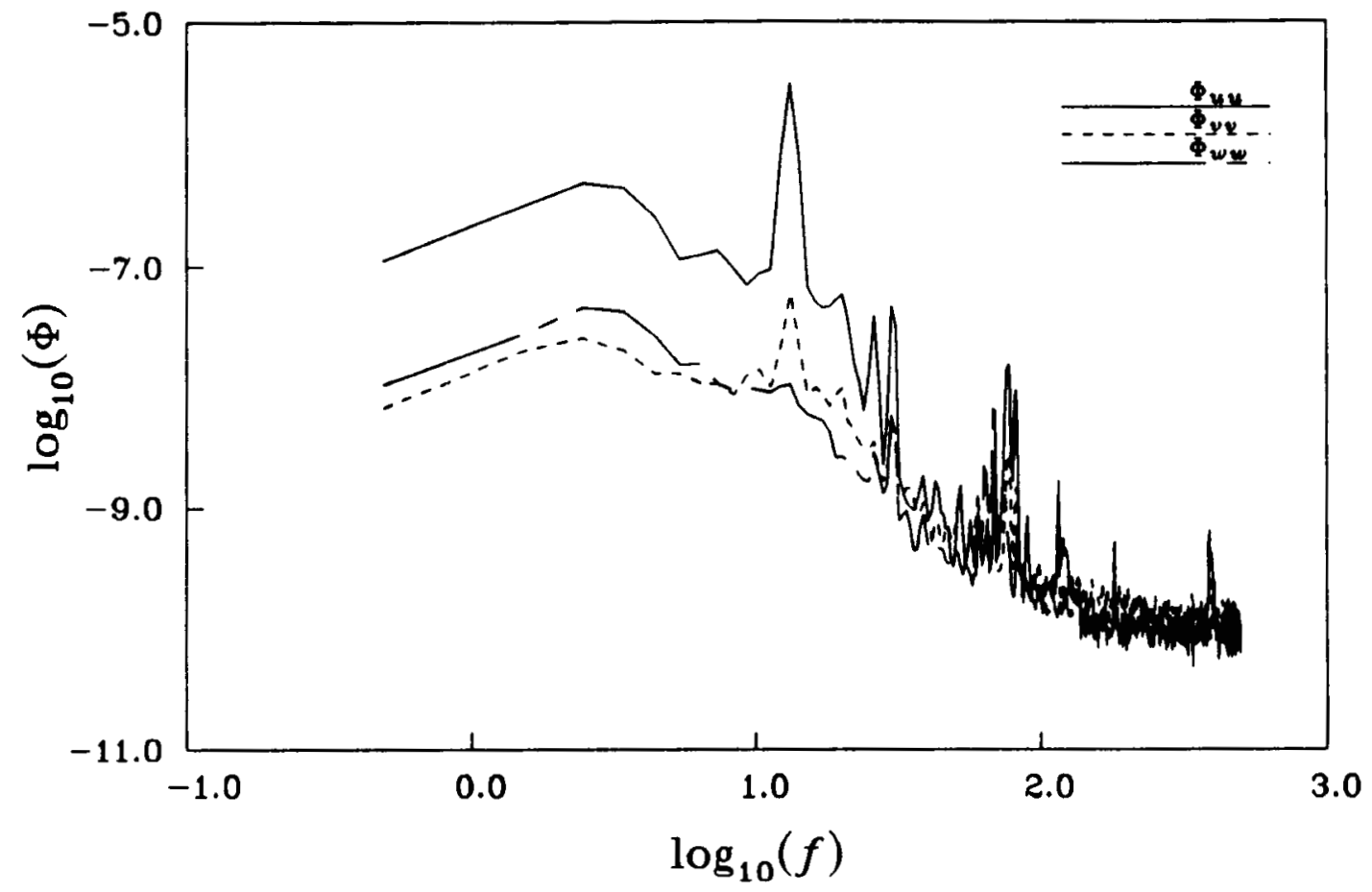

Figure 8. - Spectral densities for the three velocity components: $X=0, Y=10 \mathrm{~cm}$. Frequency increment: $0.49 \mathrm{~Hz}$.

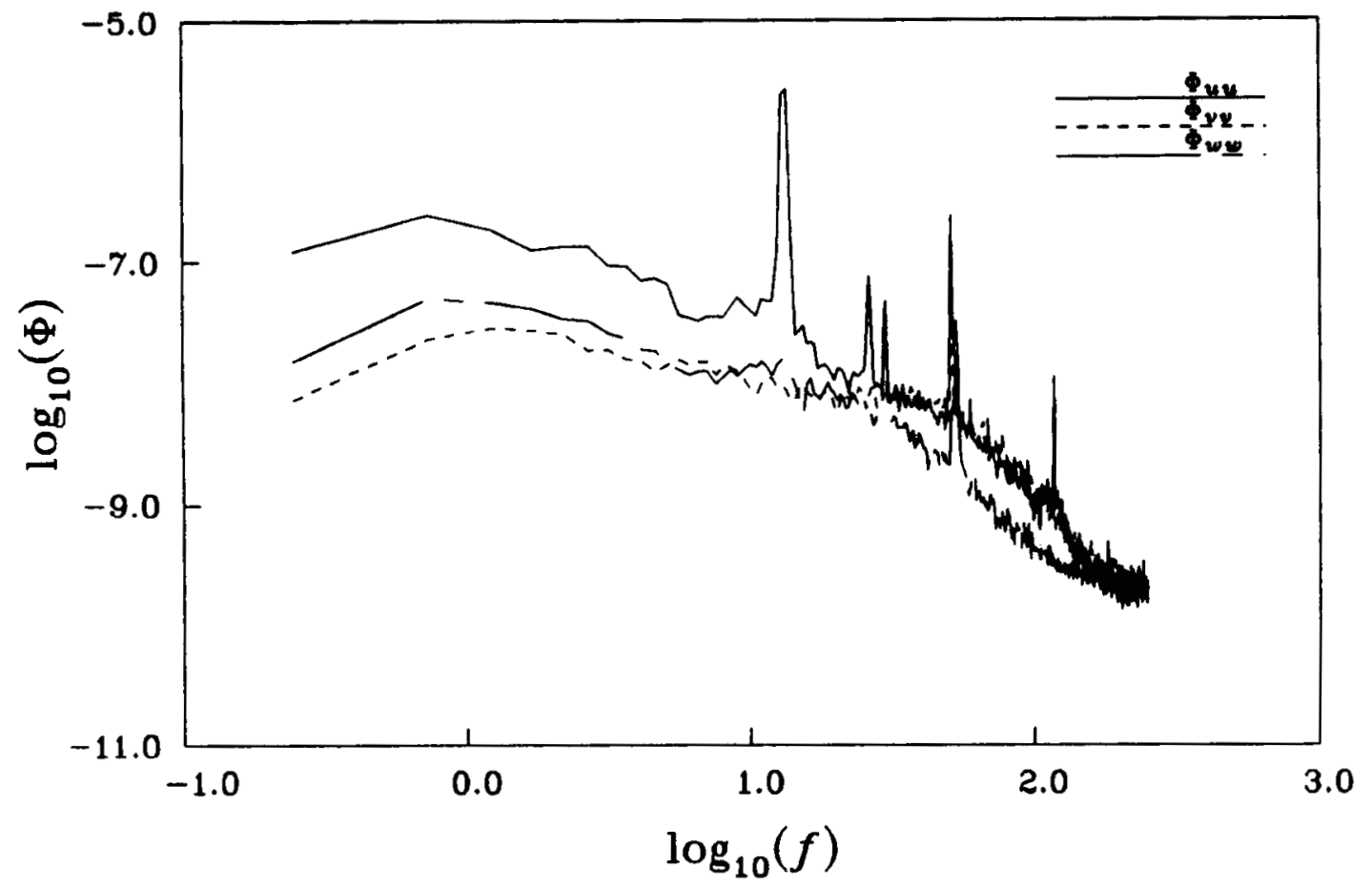

Figure 9. - Spectral densities for the three velocity components: $X=298, Y=10 \mathrm{~cm}$. Frequency increment: $0.49 \mathrm{~Hz}$. 


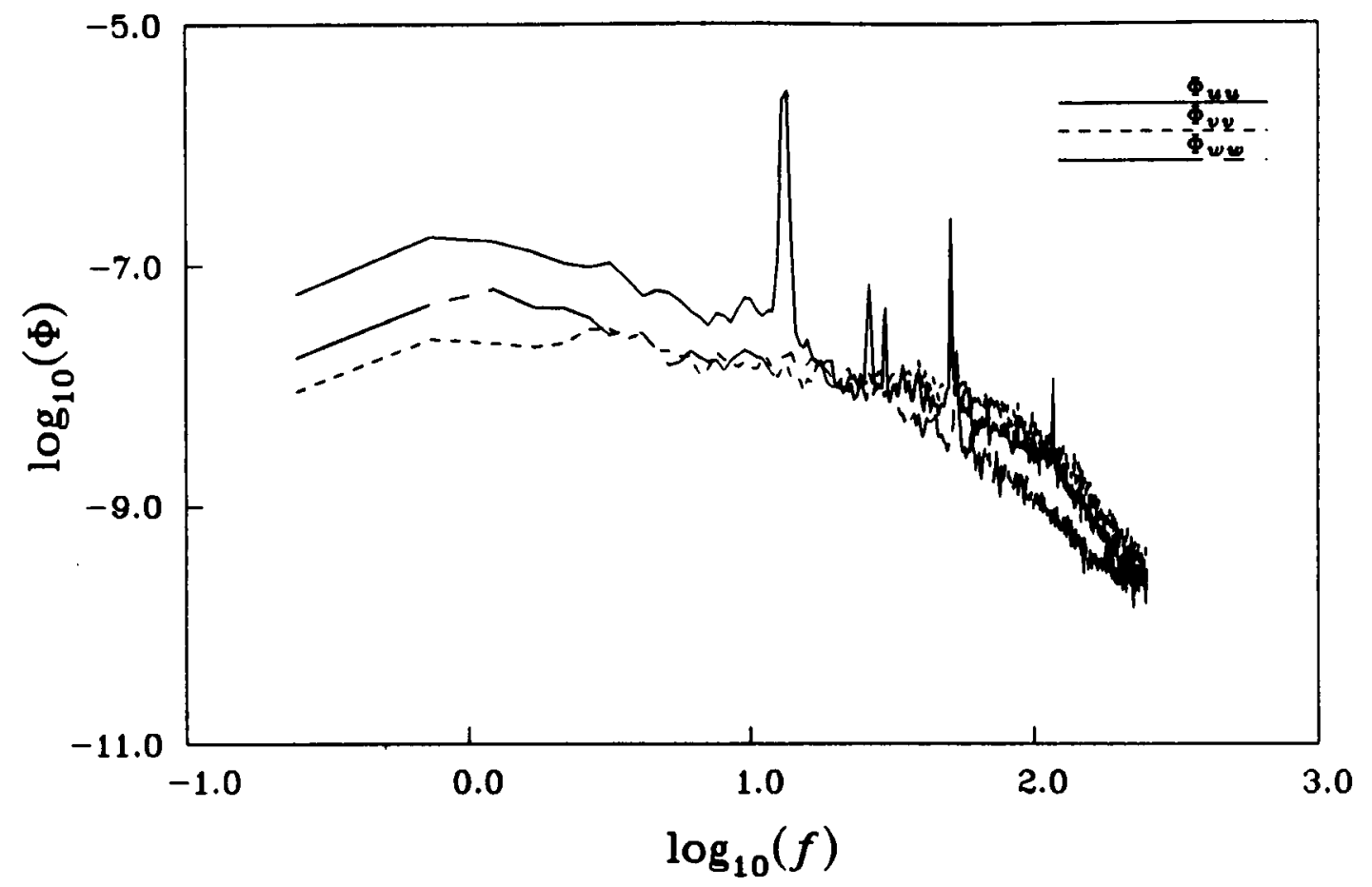

Figure 10. - Spectral densities for the three velocity components: $X=298, Y=8 \mathrm{~cm}$. Frequency increment: $0.49 \mathrm{~Hz}$.

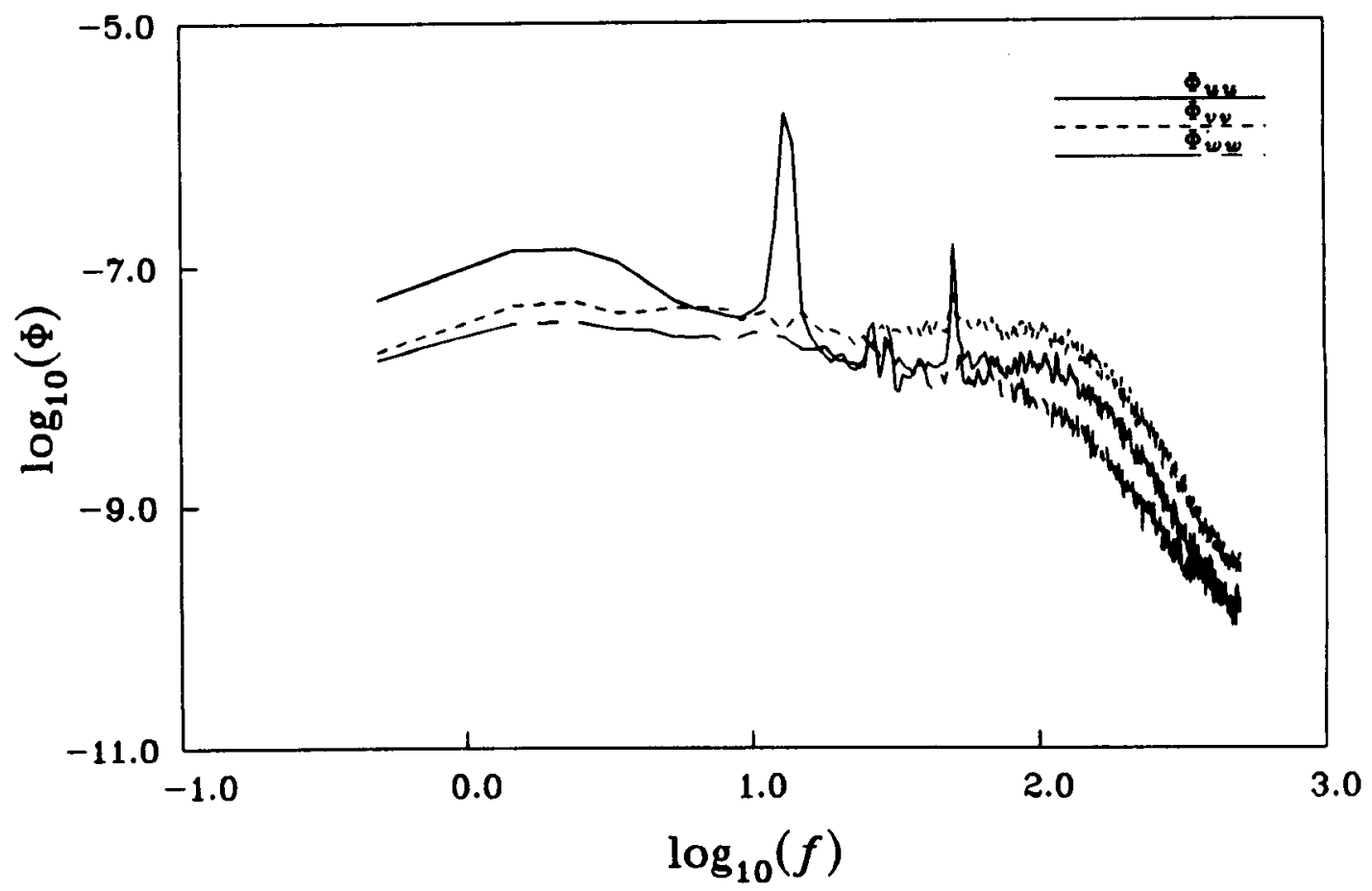

Figure 11. - Spectral densities for the three velocity components: $X=298, Y=6 \mathrm{~cm}$. Frequency increment: $0.98 \mathrm{~Hz}$. 


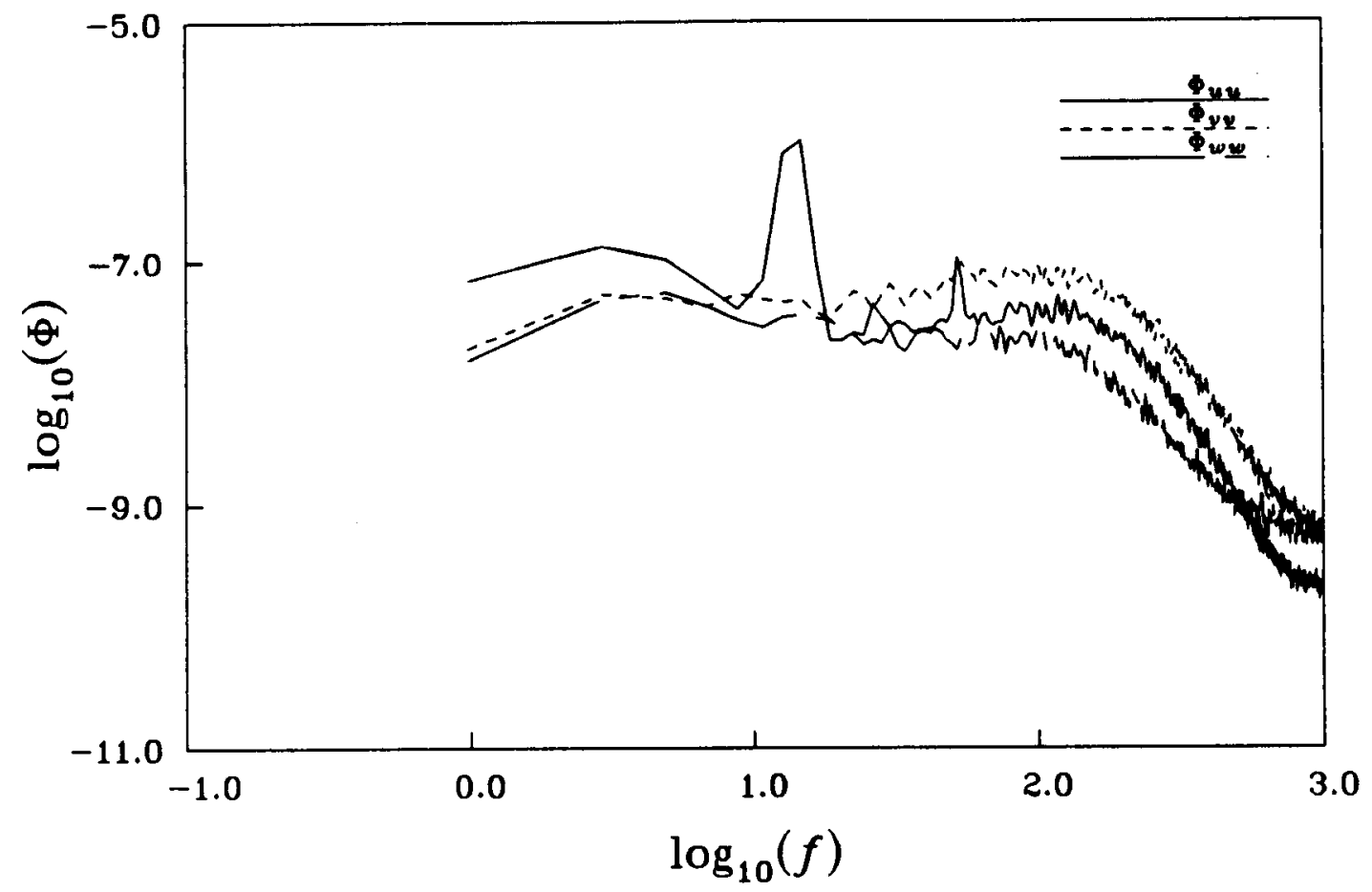

Figure 12. - Spectral densities for the three velocity components: $X=298, Y=5 \mathrm{~cm}$. Frequency increment: $1.95 \mathrm{~Hz}$.

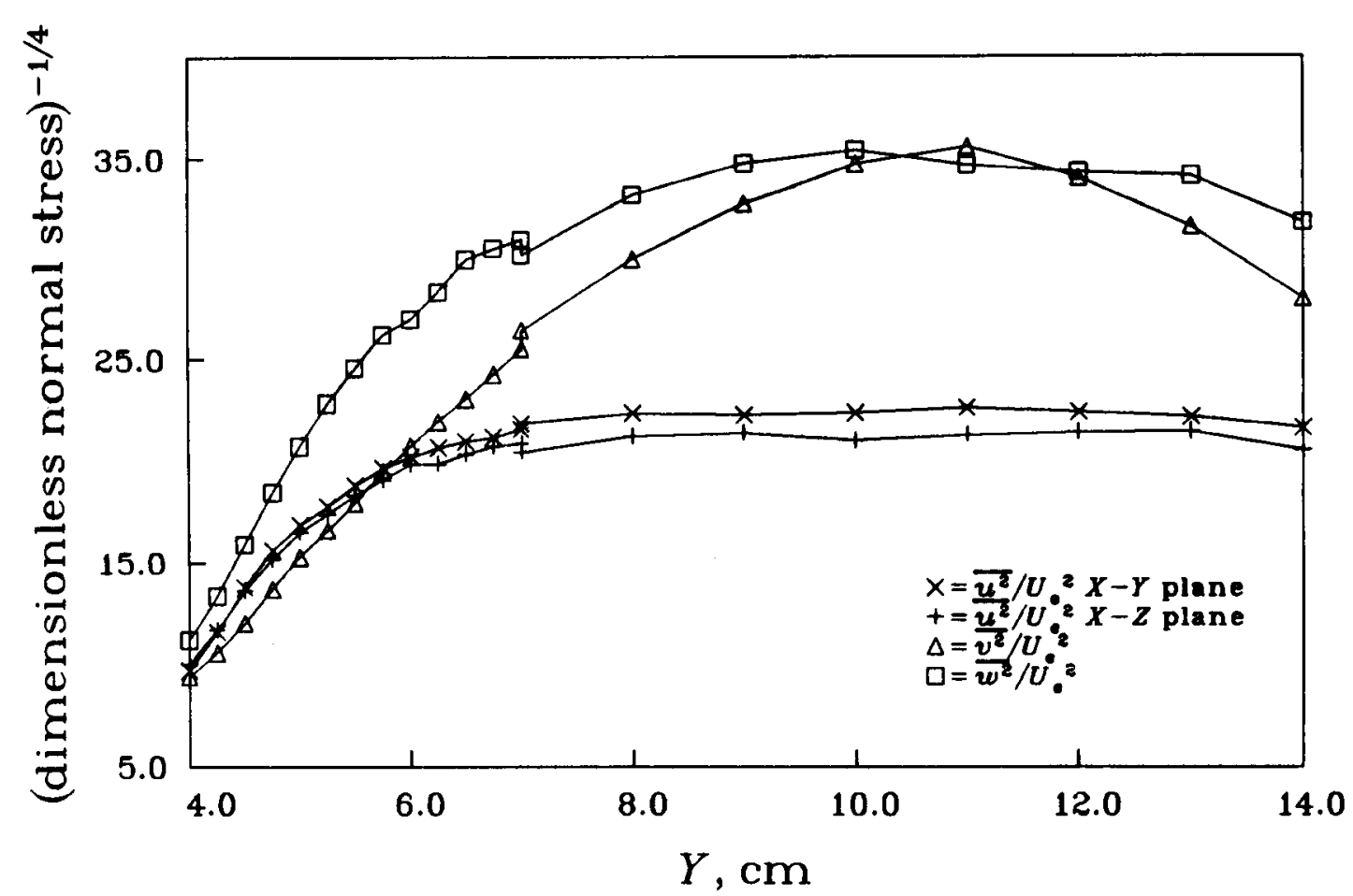

Figure 13. - Test of Phillips's theory at $X=298 \mathrm{~cm}$. 


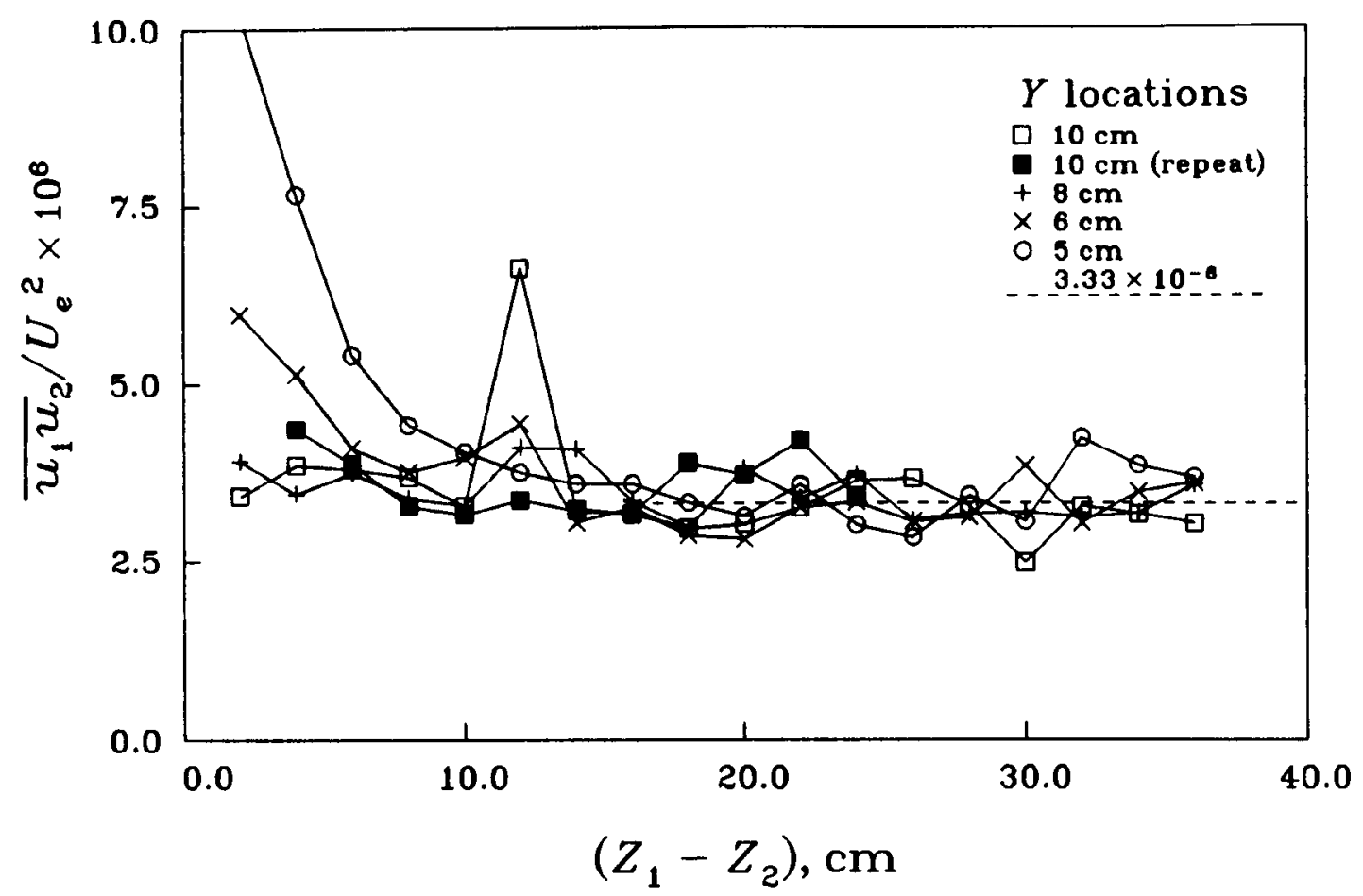

Figure 14. - Two-point covariances of single-wire measurements at $X=298 \mathrm{~cm}$.

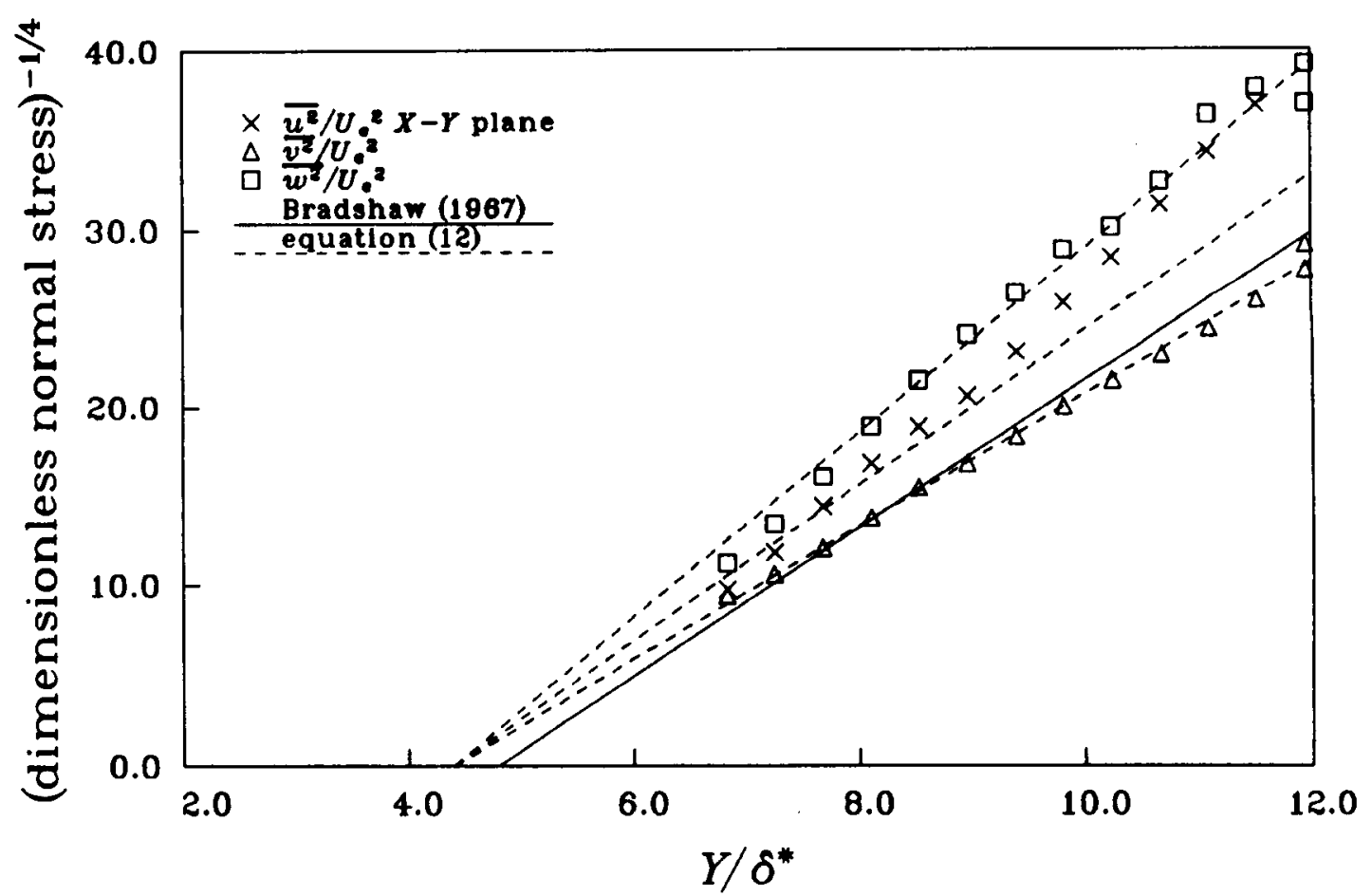

Figure 15. - Induced fluctuations near the boundary layer at $X=298 \mathrm{~cm}$. 


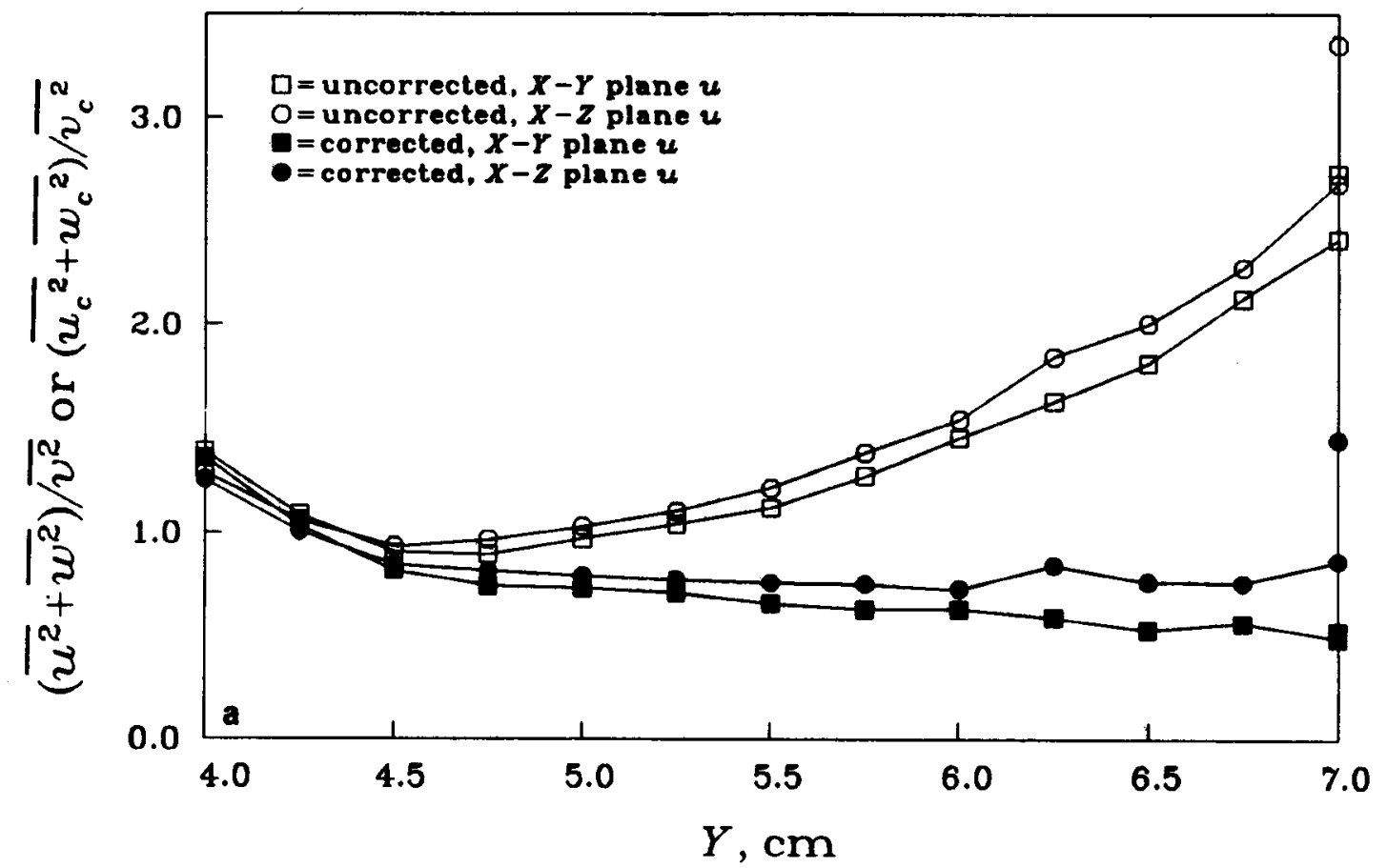

Figure 16. - Stress ratio near the boundary layer at $X=298 \mathrm{~cm}$.

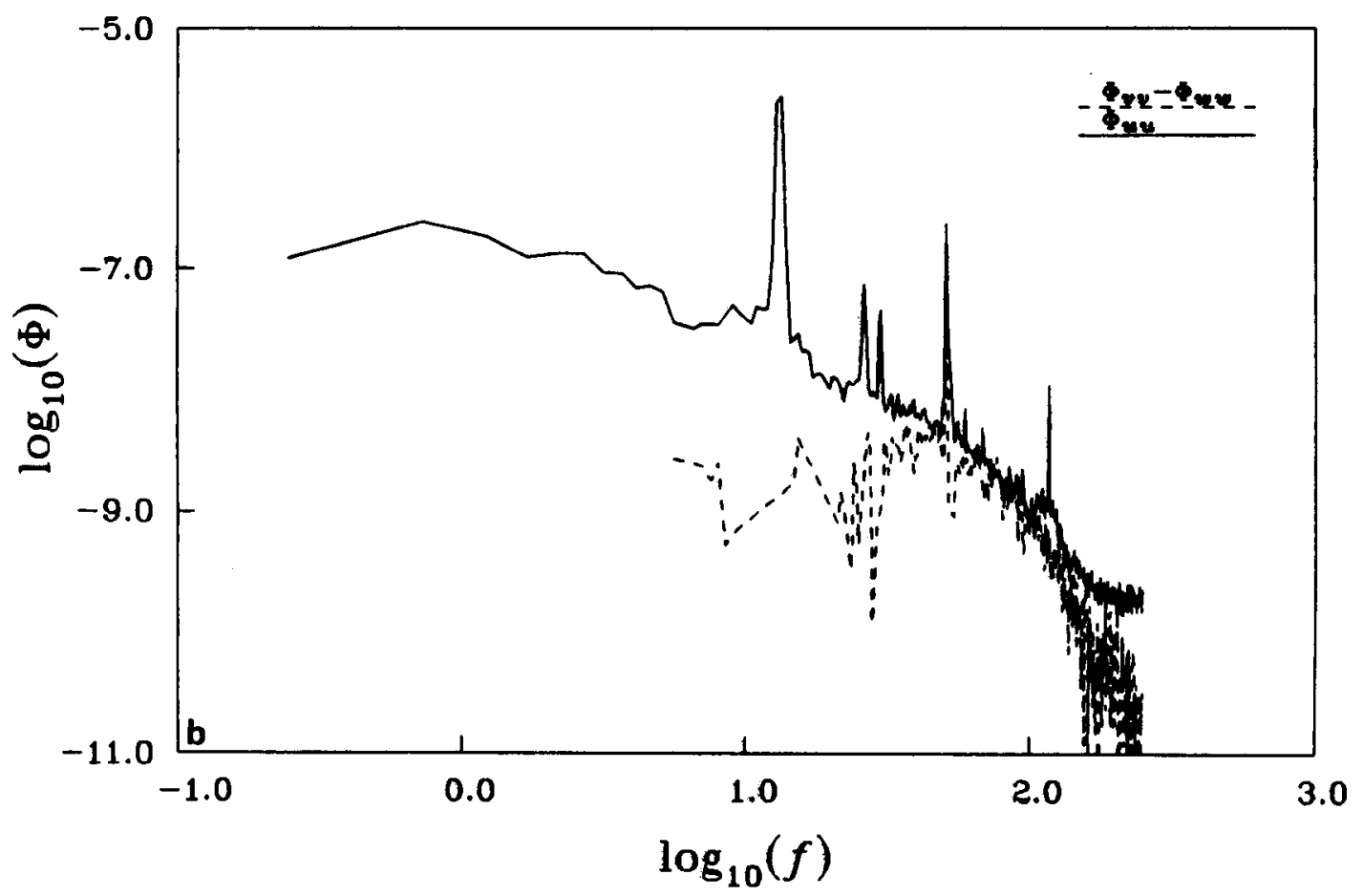

Figure 17. - Test of spectral subtraction at $X=298$; (a) $Y=10 \mathrm{~cm}$, (b) $Y=8 \mathrm{~cm}$, (c) $Y=6 \mathrm{~cm}$. 

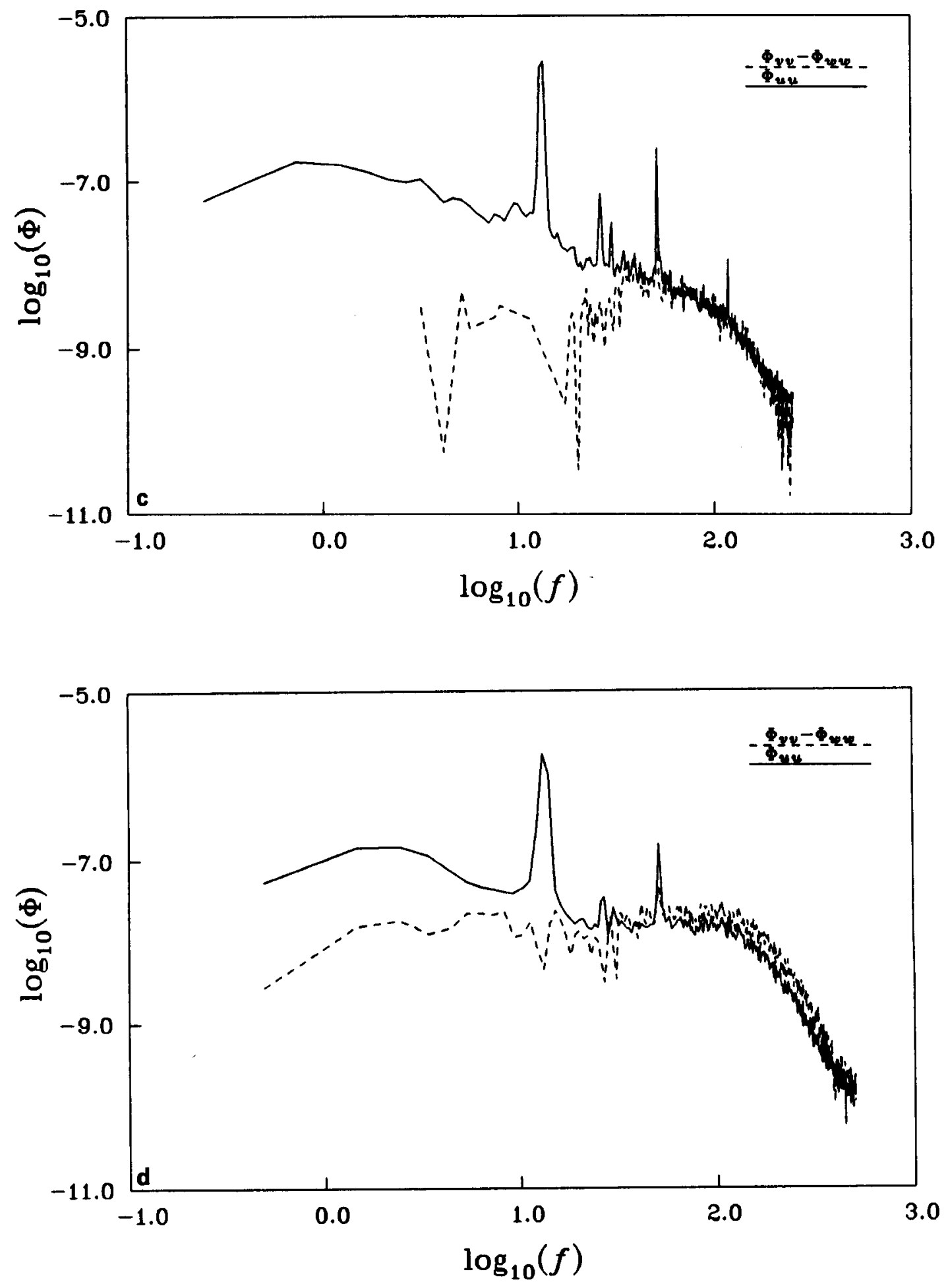

Figure 17. - concluded. 


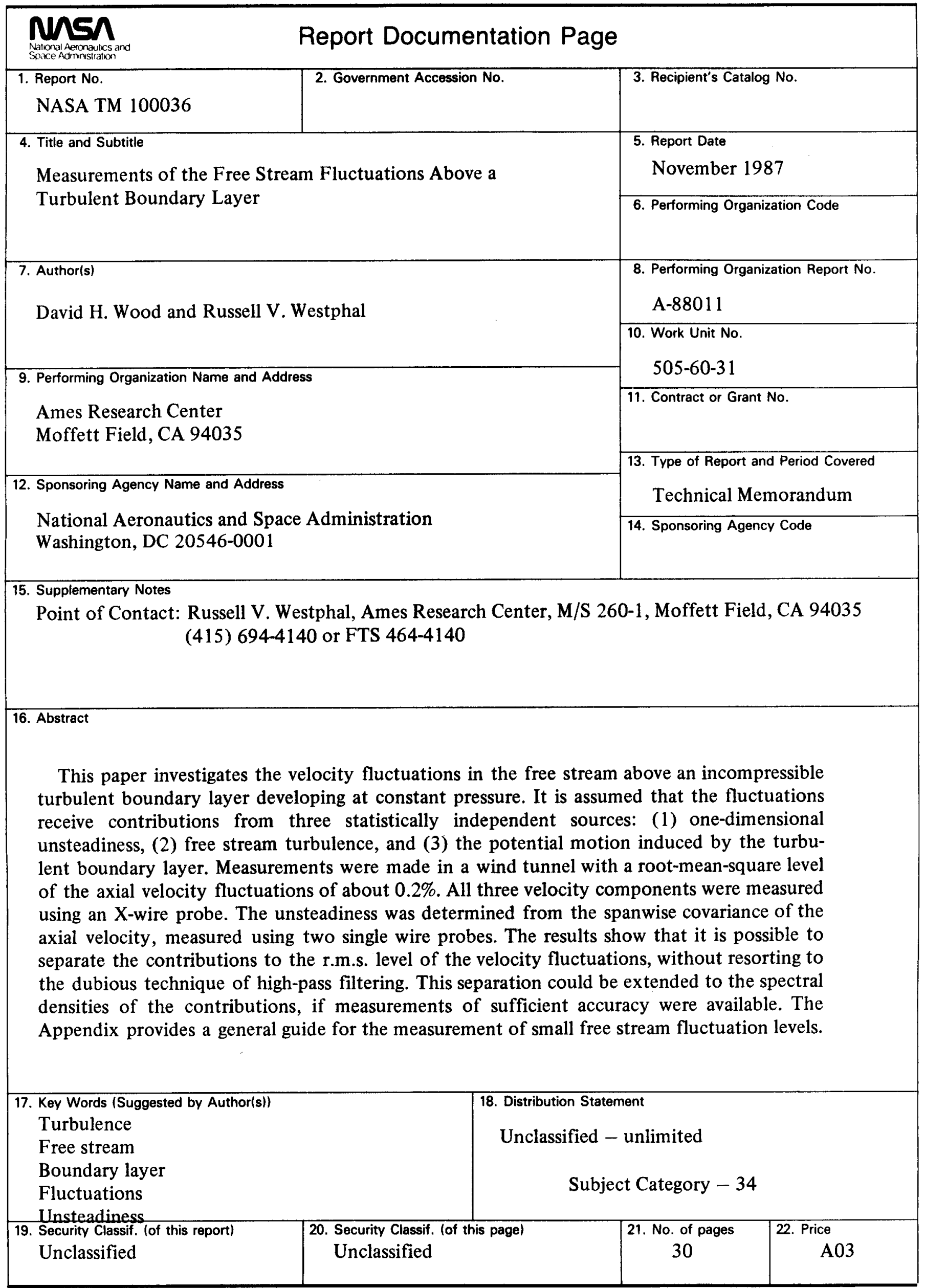

